\title{
Prolate rotation and metallicity gradient in the transforming dwarf galaxy Phoenix
}

\author{
Nikolay Kacharov, ${ }^{1 \star}$ Giuseppina Battaglia, ${ }^{2,3}$ Marina Rejkuba, ${ }^{4,5}$ Andrew A. Cole, ${ }^{6}$ \\ Ricardo Carrera, ${ }^{2,3}$ Filippo Fraternali, ${ }^{7}$ Mark I. Wilkinson, ${ }^{8}$ Carme G. Gallart, ${ }^{2,3}$ \\ Mike Irwin ${ }^{9}$ and Eline Tolstoy ${ }^{10}$ \\ ${ }^{1}$ Max Planck Institut für Astronomie, Königstuhl 17, D-69117 Heidelberg, Germany \\ ${ }^{2}$ Instituto de Astrofísica de Canarias, calle Vía Láctea s/n, E38205 - La Laguna (Tenerife), Spain \\ ${ }^{3}$ Universidad de La Laguna, Dpto. Astrofisica, E-38206 La Laguna, Tenerife, Spain \\ ${ }^{4}$ European Southern Observatory, Karl-Schwarzschild-Str. 2, D-85748 Garching bei München, Germany \\ ${ }^{5}$ Excellence Cluster Universe, Boltzmannstr. 2, D-85748 Garching bei München, Germany \\ ${ }^{6}$ School of Physical Sciences, University of Tasmania, Private Bag 37, Hobart, 7001 TAS, Australia \\ ${ }^{7}$ Department of Astronomy, University of Bologna, via Ranzani 1, I-40127 Bologna, Italy \\ ${ }^{8}$ Department of Physics and Astronomy, University of Leicester, University Road, Leicester LE1 7RH, UK \\ ${ }^{9}$ Institute of Astronomy, Madingley Road, Cambridge CB3 OHA, UK \\ ${ }^{10}$ Kapteyn Astronomical Institute, University of Groningen, Landleven 12, NL-9747AD Groningen, the Netherlands
}

Accepted 2016 December 2. Received 2016 November 25; in original form 2016 October 17

\begin{abstract}
Transition type dwarf galaxies are thought to be systems undergoing the process of transformation from a star-forming into a passively evolving dwarf, which makes them particularly suitable to study evolutionary processes driving the existence of different dwarf morphological types. Here we present results from a spectroscopic survey of $\sim 200$ individual red giant branch stars in the Phoenix dwarf, the closest transition type with a comparable luminosity to 'classical' dwarf galaxies. We measure a systemic heliocentric velocity $V_{\text {helio }}=-21.2 \pm$ $1.0 \mathrm{~km} \mathrm{~s}^{-1}$. Our survey reveals the clear presence of prolate rotation that is aligned with the peculiar spatial distribution of the youngest stars in Phoenix. We speculate that both features might have arisen from the same event, possibly an accretion of a smaller system. The evolved stellar population of Phoenix is relatively metal-poor $(<[\mathrm{Fe} / \mathrm{H}]>=-1.49 \pm 0.04 \mathrm{dex})$ and shows a large metallicity spread $\left(\sigma_{[\mathrm{Fe} / \mathrm{H}]}=0.51 \pm 0.04 \mathrm{dex}\right)$, with a pronounced metallicity gradient of $-0.13 \pm 0.01 \mathrm{dex} \operatorname{arcmin}^{-1}$ similar to luminous, passive dwarf galaxies. We also report a discovery of an extremely metal-poor star candidate in Phoenix and discuss the importance of correcting for spatial sampling when interpreting the chemical properties of galaxies with metallicity gradients. This study presents a major leap forward in our knowledge of the internal kinematics of the Phoenix transition type dwarf galaxy and the first wide area spectroscopic survey of its metallicity properties. A table containing the measured velocities, metallicities, and CaT equivalent widths of all spectroscopic targets is available online at the CDS.
\end{abstract}

Key words: techniques: spectroscopic-galaxies: dwarf-galaxies: kinematics and dynamics - Local Group-galaxies: stellar content.

\section{INTRODUCTION}

Understanding the properties of dwarf galaxies is important not only to put them in their proper cosmological context, but also to understand the formation and evolution of the most common type of galaxies.

Local Group dwarf galaxies can be divided into two main classes: gas-rich systems (called 'dIrrs' when forming stars, due to the irregular optical appearance caused by patches of recent star formation) and gas-deficient, passively evolving ones ('classical dwarf spheroidals' or 'dwarf ellipticals', at the bright end; 'ultra-faint' at the low*luminosity end). While the former are typically found in

^E-mail: kacharov@mpia.de 
isolation, the latter are in general satellites of the large Local Group spirals.

The fact that Local Group gas-rich and passive dwarfs share similar scaling relations (see e.g. Mateo 1998; Tolstoy, Hill \& Tosi 2009), appear to follow the same stellar mass-metallicity relation (Kirby et al. 2013) - but inhabit clearly different environments - already hints to similar formation mechanisms, albeit with environmental effects being relevant for the evolution of these galaxies.

To what extent and in what way dwarf galaxies are shaped by environment, it is still matter of debate. Theoretical studies show that strong tidal interactions with the host galaxy, coupled to rampressure stripping, could even turn a rotationally supported, gas-rich discy dwarf galaxy into a non-rotating, gas-deficient spheroidal system (e.g. Mayer et al. 2006). If such 'tidal stirring' is what produced the observed morphology-density relation, then the present-day structural, kinematic and dark matter properties of passive dwarfs might be considerably different than prior to their interaction with the host. On the other hand, on the basis of lifetime star formation histories from very deep colour-magnitude diagrams (CMDs), Gallart et al. (2015) propose that the environment at birth is what sets dwarf galaxies on a given evolutionary path.

Detailed studies of the kinematic and metallicity properties of the various types of dwarf galaxies inhabiting high- and low-density environments are clearly crucial for determining what properties are a common characteristic of this galaxy population and which ones are externally induced. To date, the large-scale kinematic and metallicity properties of the majority of Milky Way (MW) 'classical' dwarf spheroidal galaxies (dSphs) have been studied in detail thanks to wide-area studies based on samples of hundreds of individual stars (e.g. to mention a few, Kleyna et al. 2002; Tolstoy et al. 2004; Wilkinson et al. 2004; Coleman et al. 2005; Muñoz et al. 2005; Battaglia et al. 2006; Koch et al. 2006; Walker et al. 2006; Faria et al. 2007; Koch et al. 2007, 2008; Battaglia et al. 2008b; Walker et al. 2009; Battaglia et al. 2011; Hendricks et al. 2014a,b; Walker, Olszewski \& Mateo 2015). The star formation and chemical enrichment history of MW dSphs vary spatially within these small galaxies (see also de Boer et al. 2012a,b), so that it is clear that MW dSphs cannot be defined by one number for their age or metallicity as was initially thought. It is still to be determined though whether the observed age and metallicity gradients are caused by internal or external effects.

Gathering similarly large, spatially extended samples for the isolated Local Group dwarfs, as well as M31 satellites, is more challenging and time consuming due to their larger heliocentric distances. Nevertheless, notable efforts in this direction have recently taken place (e.g. Ho et al. 2012; Collins et al. 2013; Kirby et al. 2013, 2014). In particular, both the kinematics and metallicity properties of WLM, a dwarf irregular (dIrr) galaxy at the edge of the Local Group, has been extensively studied from a sample of 200 red giant branch (RGB) stars. This dIrr has a shallower metallicity gradient when compared with most well-studied MW dSphs, but similar to those of larger and rotation supported systems as the Large and Small Magellanic Clouds (Leaman et al. 2013), suggesting that mass and angular momentum might play a role in driving the presence/absence of metallicity gradients (Schroyen et al. 2011).

While it had previously been thought that dSphs were exclusively pressure-supported, hints of rotation were found in the outer parts of the MW dSph Sculptor (Battaglia et al. 2008a), as well as in isolated dSphs (Cetus: Lewis et al. 2007; Tucana: Fraternali et al. 2009). Overall though, the stellar component of most Local Group dwarf galaxies studied so far is found to be dispersion-supported, possibly independently on morphological type and environment (Wheeler et al. 2015). Even though sample sizes and spatial coverages of current spectroscopic samples of individual stars in Local Group dwarfs are rather inhomogeneous, this result suggests that most dwarf galaxies form as thick, dispersion-supported systems and if passively evolving dwarfs originate from gas-rich systems, such transformation may occur just by removal of the gaseous component. Interestingly, so far, only one Local Group dwarf galaxy, the And II dSph, has been found to display prolate rotation (Ho et al. 2012), attributed to a merger with another small galactic system (Amorisco, Evans \& van de Ven 2014; Łokas et al. 2014).

In this paper, we focus on the Phoenix (Phx) dwarf galaxy that belongs to the handful of Local Group dwarf galaxies displaying intermediate properties between dIrrs and dSphs (and therefore called 'transition type dwarfs', dIrr/dSph). These are particularly interesting objects since they are thought to be in the process of losing their gas and therefore about to evolve into passive systems. Being directly comparable to classical dSphs in terms of luminosities and sizes but typically found in less dense environments, dIrr/dSph offer the possibility of looking at the physical characteristics of dSphslike systems in absence of strong interactions with large galaxies.

Located at $\sim 450 \mathrm{kpc}$ from the Sun (van de Rydt, Demers \& Kunkel 1991), Phx is the closest dIrr/dSph and therefore a good candidate for accurate studies. It is found outside the virial radius of the MW and M 31, hence arguably environmental effects were not the main driver in its evolution. Phx has a luminosity similar to the Sculptor dSph $\left(L_{\mathrm{V}}=0.9 \times 10^{6}\right.$ and $2.15 \times 10^{6} \mathrm{~L} \odot$, respectively; Mateo 1998) and, like Sculptor, it formed most of its stars more than 10 Gyr ago (Hidalgo et al. 2009). However, unlike Sculptor, $\mathrm{Phx}$ has been forming stars recently, up until $100 \mathrm{Myr}$ ago (Young et al. 2007). This shows that Phx was typically able to retain some gas, probably because of its larger distance from the MW and/or a larger total mass.

To date, spectroscopic studies of resolved stars in Phx have been scarce. Even its optical systemic velocity is uncertain, with conflicting results from two spectroscopic studies based on only a few RGB stars in the central region of Phx $\left(-52 \pm 6 \mathrm{~km} \mathrm{~s}^{-1}\right.$ : Gallart et al. $2001 ;-13 \pm 9 \mathrm{~km} \mathrm{~s}^{-1}$ : Irwin \& Tolstoy 2002). This makes the physical association with a nearby $\mathrm{H}$ I cloud (Young \& Lo 1997; St-Germain et al. 1999) still debatable. The available photometry shows that the young stars are centrally concentrated and hints at differences in the spatial distribution of the older and intermediate-age stars, as observed for several dSphs (Battaglia et al. 2012; Hidalgo et al. 2013). In addition, the spatial distribution of the young stars is tilted by $\sim 90^{\circ}$ with respect to $\mathrm{Phx}$ main body and aligned with the projected minor axis (Martínez-Delgado, Gallart \& Aparicio 1999a; Hidalgo et al. 2009; Battaglia et al. 2012). Interestingly, signs of kinematic properties varying with position angle have been reported in a conference proceeding by Zaggia et al. (2011). Nothing is known about the metallicity properties of this galaxy nor whether it is rotationally or pressure-supported.

Here we extend to Phx the successful approach applied to MW dSphs, by deriving its large-scale metallicity and kinematic properties from a wide-area spectroscopic survey of $~ 200$ RGB stars in the $\mathrm{Ca}$ II triplet (CaT) region. In Sections 2 and 3, we present the data set, the data reduction procedure and the determination of metallicities $([\mathrm{Fe} / \mathrm{H}])$ from the nIR CaT lines. In Section 4 , we discuss the membership selection. Sections 5 and 6 contain the analysis of the internal kinematic and metallicity properties. Finally, we conclude and summarize the main results in Section 7. The Phx parameters adopted throughout this work are summarized in Table 1 . 
Table 1. Adopted parameters for Phx.

\begin{tabular}{lcl}
\hline Parameter & Value & \multicolumn{1}{c}{ Ref. } \\
\hline$\alpha_{\mathrm{J} 2000}$ & $01^{\mathrm{h}} 51^{\mathrm{m}} 06^{\mathrm{s}}$ & Mateo (1998) \\
$\delta_{\mathrm{J} 2000}$ & $-44^{\circ} 26^{\prime} 41^{\prime \prime}$ & Mateo (1998) \\
Ellipticity $^{a}$ & 0.3 & Battaglia et al. (2012) \\
$\mathrm{PA}$ & $5^{\circ}$ & Battaglia et al. (2012) \\
$R_{\text {core }}$ & $1.79 \operatorname{arcmin}$ & Battaglia et al. (2012) \\
$R_{\text {tidal }}$ & 10.56 arcmin & Battaglia et al. (2012) \\
$R_{1 / 2}$ & 2.30 arcmin & Battaglia et al. (2012) \\
$R_{\mathrm{S}}$ & 1.67 arcmin & Battaglia et al. (2012) \\
$m$ & 0.88 & Battaglia et al. $(2012)^{b}$ \\
$V_{H B}$ & 23.9 & Holtzman et al. $(2000)$ \\
$\mathrm{dm}_{0}$ & $23.09 \pm 0.10$ & McConnachie $(2012)$ \\
$E(B-V)$ & 0.016 & McConnachie (2012) \\
\hline
\end{tabular}

Notes. ${ }^{a} e=1-\frac{b}{a}$.

${ }^{b}$ We re-derived the Sérsic profile parameters (characteristic radius $R_{\mathrm{S}}$ and power $m$ ) since we noticed that some duplicates were present in the 2012 photometry in the overlapping regions between the different pointings. Removing these duplicates from the photometric catalogue does not lead to any significant changes in terms of structural properties.

\section{OBSERVATIONS AND DATA REDUCTION}

Observations have been taken with the FORS2 instrument in multiobject spectroscopic mode with exchangeable masks. FORS2 is a focal reducer and visual-light multipurpose instrument that offers imaging, polarimetry, low-dispersion long-slit and multi-object spectroscopy (Appenzeller et al. 1998) mounted on the Very Large Telescope (VLT) at the European Southern Observatory (ESO) Paranal Observatory.

The bulk of our analysis is based on a data set taken in service mode between 2009 September 18 and 29 within ESO Programme ID 083.B-0252(B) (PI: Battaglia), when FORS2 was mounted at the Cassegrain focus of Antu (UT1). Within this programme, we obtained spectra of 254 stars with $I<21.5$ mag distributed over 11 different pointings, covering a total area of $\sim 10 \operatorname{arcmin} \times 20 \mathrm{arcmin}$ across the galaxy (Fig. 1). 11 stars were targeted more than once in different masks and could thus be used to assess the measurement errors from independent observations.

This main data set is complemented with another one taken in visitor mode on 2003 August 23 within ESO Programme ID 71.B0516 (PI: Cole), when FORS2 was mounted at the Cassegrain focus of Yepun (UT4). We observed one position in central Phx targeting 40 stars within 1 mag of the tip of the RGB $(19.2 \lesssim I \lesssim 20)$. There are 14 stars in common between the two data sets. Both data sets are analysed and presented here for the first time.

The observing set-up was identical during both runs: we used the $1028 \mathrm{z}$ grism in combination with the OG590+32 order separation filter (wavelength coverage 7730-9480 ̊, dispersion $0.42 \AA \mathrm{px}^{-1}$, spectral resolution $R=\lambda / \Delta \lambda=2560$ ) and the standard resolution mode of the red-sensitive MIT detector (two $2 \mathrm{k} \times 4 \mathrm{k}$ px CCDs mosaic, with $2 \times 2$ binning that yields $0.25 \operatorname{arcsec} \mathrm{px}^{-1}$ spatial scale). We used the Mask eXchange Unit (MXU) mode to design 1 arcsec wide ( 10 arcsec long) slits across the 6.8 arcmin $\times 6.8$ arcmin field of view $(\mathrm{FoV})$. Targets were selected based on FORS2 pre-imaging taken in $V$ and $I$ band prior to each spectroscopic run. The preimaging observations used for the selection of the spectroscopic targets for the programme ID 083.B-0252 (Period 83 or P83) are described in Battaglia et al. (2012). The priority was given to targets identified along the Phx RGB (Fig. 2), with few stars added outside this colour range, when the slits could not be allocated to a suitable Phx RGB candidate.

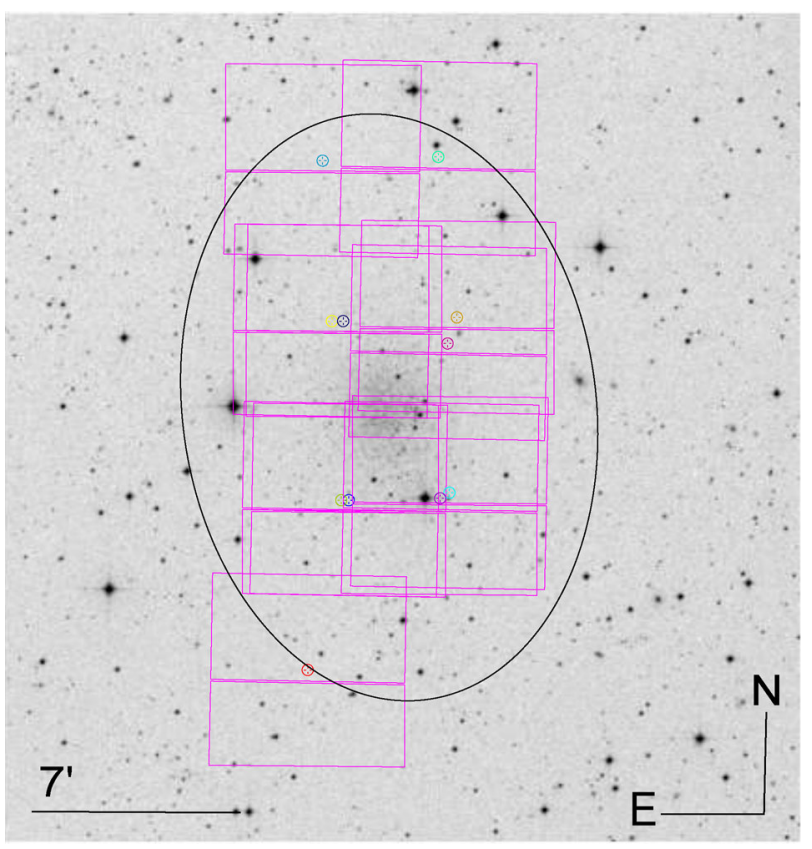

Figure 1. DSS image of the Phx dwarf galaxy. The field of view of FORS2 in MXU mode is overplotted for the 11 pointings from the P83 observing run. The centres of the different pointings are marked with different colour symbols. The ellipse shows the nominal tidal radius, position angle and ellipticity of Phx (Battaglia et al. 2012).

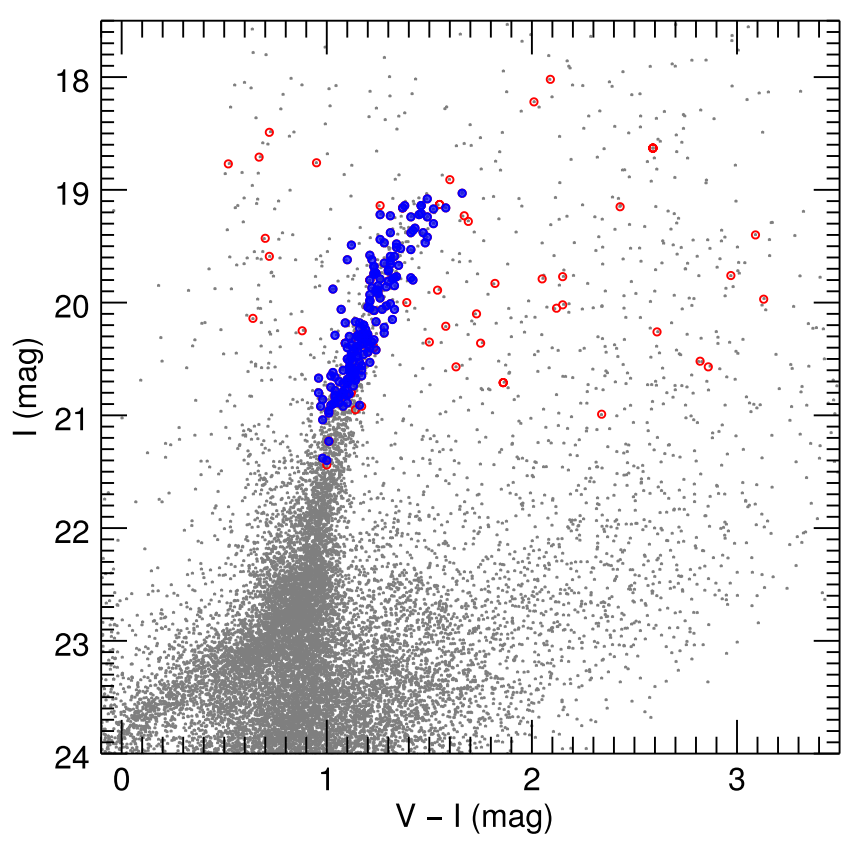

Figure 2. CMD of the Phx dwarf galaxy from VLT/FORS2 photometry (Battaglia et al. 2012). The FORS2 MXU targets are marked with open, red circles and the probable Phx member stars are shown with solid, blue symbols.

In addition to Phx stars, observations were also taken with MXU masks designed to target RGB stars in Galactic globular clusters in order to calibrate the relationship between $\mathrm{CaT}$ equivalent width (EW) and metallicity (Leaman et al. 2009; Swan et al. 2016). We observed two clusters that bracket the expected metallicity range of 
Table 2. Observing log.

\begin{tabular}{lcccc}
\hline Field $^{a}$ & $\begin{array}{c}\text { RA2000 } \\
\left({ }^{\circ}\right)\end{array}$ & $\begin{array}{c}\text { DEC2000 } \\
\left({ }^{\circ}\right)\end{array}$ & $\begin{array}{c}\text { Date } \\
\text { 2009 September }\end{array}$ & $\begin{array}{c}\text { Exp. time } \\
(\mathrm{s})\end{array}$ \\
\hline Phx-14 & 27.84209 & -44.60355 & $27 \& 28$ & $2 \times 2730$ \\
Phx-10-1 & 27.80934 & -44.50062 & $21 \& 25$ & $3 \times 2730$ \\
Phx-10-2 & 27.81683 & -44.50057 & 23 & $1 \times 2730$ \\
Phx-11-1 & 27.72505 & -44.49538 & 26 & $3 \times 2730$ \\
Phx-11-2 & 27.73237 & -44.49931 & 18 & $2 \times 2730$ \\
Phx-06-1 & 27.82667 & -44.39353 & 26 & $3 \times 2730$ \\
Phx-06-2 & 27.81574 & -44.39362 & 22 & $2 \times 2730$ \\
Phx-07-1 & 27.72061 & -44.39015 & $24 \& 25$ & $4 \times 2730$ \\
Phx-07-2 & 27.72773 & -44.40565 & $27 \& 28$ & $2 \times 2730$ \\
Phx-02 & 27.83579 & -44.29739 & 27 & $1 \times 2730$ \\
Phx-03 & 27.73828 & -44.29482 & 29 & $1 \times 2730$ \\
47 Tuc & 5.65952 & -72.07518 & 23 & $3 \times 100$ \\
M 15 & 322.53856 & +12.17369 & 24 & $3 \times 300$ \\
& & & 2003 August & \\
Phx (centre) & 27.76975 & -44.42647 & 23 & $6 \times 2000$ \\
\hline
\end{tabular}

${ }^{a}$ Fields are ordered from south to north and east to west.

Phx: 47 Tuc, $[\mathrm{Fe} / \mathrm{H}]=-0.72 \mathrm{dex}$ and $\mathrm{M} \mathrm{15},[\mathrm{Fe} / \mathrm{H}]=-2.37 \mathrm{dex}$ (Harris 1996, 2010 edition $^{1}$ ), each with one MXU mask per cluster.

The observing logs including number of exposures and exposure times for each pointing and the central pointing coordinates for 2009 (referred to as P83 according to ESO parlance) and 2003 (referred to as P71 run below) observing runs are in Table 2.

In the following sections we describe our data reduction procedure and radial velocity measurements, emphasizing in particular (small) differences between the two data sets and the strategy adopted to produce the final homogeneous sample used for the kinematic and metallicity analysis.

\subsection{Data reduction}

Data reduction and extraction of the spectra was performed within IRAF. $^{2}$ Using standard IRAF procedures, master bias and biassubtracted, normalized master flat-field images, were created from standard calibrations (five frames each for bias and lamp flats) taken typically in the morning following the science observations. These master calibrations were used to correct the two-dimensional (2D) science and wavelength calibration exposures. We then applied the spectroscopic modification of the L. A. Cosmic ${ }^{3}$ algorithm (van Dokkum 2001) to remove cosmic rays from the science frames.

At this point, only the individual exposures of each of the two calibration globular clusters masks were median combined. Due to the short interval of time in which these exposures were recorded, there were practically no shifts nor tilts among them. This was, however, not the case for the Phx exposures, where some shifts and PSF variations due to changing seeing over the night or between different nights were evident.

We used custom designed IRAF scripts to trace the apertures and correct for distortions the science frames and associated arc-lamp frames. Data taken between 2009 September 18 and 28 showed strongly tilted spectra both in calibration (arc-lamp) and science

\footnotetext{
${ }^{1}$ http://www.physics.mcmaster.ca/resources/globular.html

2 IRAF is distributed by the National Optical Astronomy Observatories that are operated by the Association of Universities for Research in Astronomy, Inc., under cooperative agreement with the National Science Foundation. http://iraf.noao.edu/

${ }^{3}$ http://www.astro.yale.edu/dokkum/lacosmic/
}

frames. The tilt was of the order of $\sim 50$ binned pixels between start and end of the CCD and was caused by grism misalignment. To correct for this, we applied the wavelength solution directly on the rectified 2D spectra before extraction. More specifically, we cut the individual rectified $2 \mathrm{D}$ spectra from the science and arc-lamp images, identified the lines from the arc-lamp with IRAF identify and re-identify tasks and then traced their position along the slit to obtain a 2D model of the wavelength calibration for each individual slit with fitscoord and transform tasks. Applying this model, we obtained wavelength calibrated 2D spectra with straightened sky lines, based on which subsequent sky-subtraction could be substantially improved. We continued with optimal extraction and sky subtraction using the standard IRAF procedure apall, which results in the optimally extracted 1D science spectrum accompanied with additional extensions that have the extracted sky spectrum and the error spectrum - the flux uncertainty at every pixel. At this point, we performed an additional refinement to the wavelength calibration using the measured positions of the sky lines in the extracted sky spectra, such that radial velocities measured from the sky spectra had a mean of $0 \pm 2 \mathrm{~km} \mathrm{~s}^{-1}$. The resulting 1D spectra were then continuum normalized using a high-order polynomial fits within the IRAF task continuum.

We note that for the P71 single MXU mask data reduction was slightly different: the extracted sky spectra were used to determine the dispersion solution of the spectra individually and then the spectra were continuum normalized, shifted to a common wavelength solution and combined to produce the final spectrum.

\subsection{Radial velocity measurements and wavelength calibration accuracy}

The good centring of the stars in the slits in low-resolution spectroscopy is crucial for the accurate determination of the radial velocities, in particular when seeing is of the order of or smaller than the slit width (which was the case for most of our observations). We performed a consistency check for the centring of each star's centroid on the slit using the through-slit images taken before the spectroscopic exposures. This showed that most masks were well centred to within $0.1 \pm 0.1 \mathrm{px}$ (median and standard deviation; $1 \mathrm{px}=28 \mathrm{~km} \mathrm{~s}^{-1}$ ). Only in two exposures of one P83 mask we measured a significant slit centre offset of $0.3 \pm 0.1 \mathrm{px}$, as well as in the two globular cluster masks $(0.7 \pm 0.2$ and $0.2 \pm 0.2 \mathrm{px}$ for 47 Tuc and M 15, respectively). Likely, the worse slit alignment of globular cluster masks was due to bright targets that appeared to fill the entire slit even with very short exposure, unless one uses additional filter or adjusts and carefully examines the cuts in the through-slit images. These exposures were corrected for the corresponding radial velocity offsets.

We measured the radial velocities from the 1D wavelengthcalibrated spectra of each individual exposure using the IRAF $f x$ cor task, which uses a Fourier cross-correlation to determine the Doppler offset of the spectrum of interest compared to a template spectrum. As a template, we used a synthetic spectrum derived from an interpolated Kurucz stellar atmospheric model and convolved to match the FORS2 spectral resolution with similar parameters as expected for the Phx targets (i.e. RGB stars of moderately low metallicity $\left.-\log g=1.0, T_{\text {eff }}=4000 \mathrm{~K},[\mathrm{Fe} / \mathrm{H}]=-1.5 \mathrm{dex}\right)$. Then we calculated the heliocentric radial velocities using the rvcorrect task in IRAF. The fxcor task also computes velocity uncertainties based on the fitted correlation peak height and the antisymmetric noise (see Tonry \& Davis 1979), which turned to have a median value of $\pm 12 \mathrm{~km} \mathrm{~s}^{-1}$. To this, we added in quadrature the 

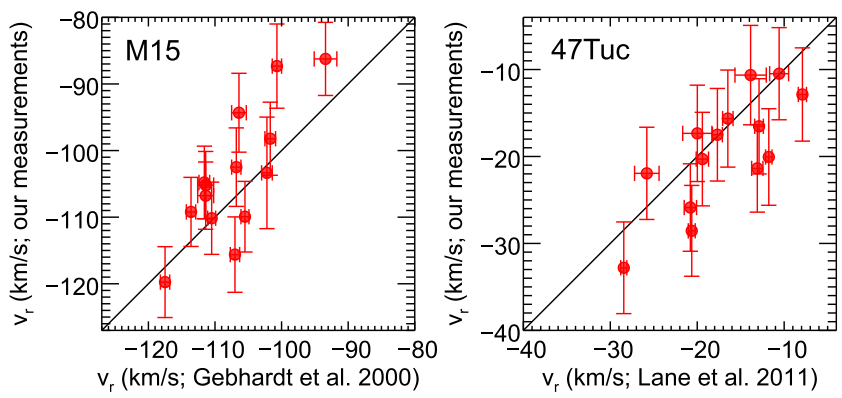

Figure 3. A comparison of our radial velocity measurements for the two globular clusters with high-resolution measurements for the same stars from Gebhardt et al. (2000, M 15) and Lane et al. (2011, 47 Tuc). The black line is not a fit, but a 1:1 relation.

velocity uncertainty due to the wavelength calibration (median value of $\left.\pm 2 \mathrm{~km} \mathrm{~s}^{-1}\right)$ and the slit centring $\left( \pm 4 \mathrm{~km} \mathrm{~s}^{-1}\right)$ systematic errors. The final velocity of each star, and the corresponding velocity uncertainty, was calculated as the weighted mean and error of the weighted mean of the velocities from the individual exposures. As discussed below, we have a better handle on the velocity uncertainties from individual spectra, rather than measuring radial velocities from the stacked spectra.

Finally, the spectra of the individual exposures were Dopplercorrected and combined using a weighted average where more than one exposure per star was available. Due to the small number of individual exposures per star (typically 2 or 3 ; see Table 2 ), a median combination was not justified. In order to obtain an error spectrum for each reduced science spectrum, the individual $\sigma$-spectra were divided by the polynomial used for the continuum normalization of the science spectra and combined using the formula for estimating the error of a weighted mean $\left(\sigma^{2}(\lambda)=1 / \Sigma\left[1 / \sigma_{i}^{2}(\lambda)\right]\right)$. The median signal-to-noise ratio (SNR) of our spectra is 23 per px for the $\mathrm{Phx}$ masks and 77 and 90 per px for 47 Tuc and M 15, respectively. We note that we use the regions between 8575 and $8630 \AA$ and 8700 and $8750 \AA$ to track the continuum level and uniformly estimate the SNR for each spectrum.

We matched the observed stars in the two globular clusters with high-resolution radial velocity catalogues (Gebhardt et al. 2000; Lane et al. 2011, for M 15 and 47 Tuc, respectively) to assess the accuracy of our measurements. The results of the comparison are presented in Fig. 3. There is no significant systematic offset between our radial velocity estimates and the results from high-resolution studies. The mean radial velocities from our FORS2 observations for M 15 and 47 Tuc are $-104.0 \pm 2.5$ and $-19.6 \pm 1.7 \mathrm{~km} \mathrm{~s}^{-1}$, respectively, in good agreement with the corresponding values published in the Harris $(1996,2010$ version) catalogue $(-107.0 \pm 0.2$ for M 15 and $-18.0 \pm 0.1 \mathrm{~km} \mathrm{~s}^{-1}$ for 47 Tuc). We note that the globular clusters spectra have significantly higher SNR, on average, than our Phx spectra and hence also smaller velocity uncertainties.

For the Phx targets, we tested the accuracy of the velocity measurements using 11 stars that were observed in more than one mask. We compared the difference between the derived velocities from each mask for these stars in common (Fig. 4) both from measurements performed on stacked spectra and on individual exposures. The estimated uncertainties in the first case (measurement from stacked spectra) are too small compared to the standard deviation of the distribution of $17 \mathrm{~km} \mathrm{~s}^{-1}$, while the median, quadrature combined error for the presented differences is $11 \mathrm{~km} \mathrm{~s}^{-1}$. On the other hand, the second case (RV measurements from individual exposures) results are in much better agreement, with the standard

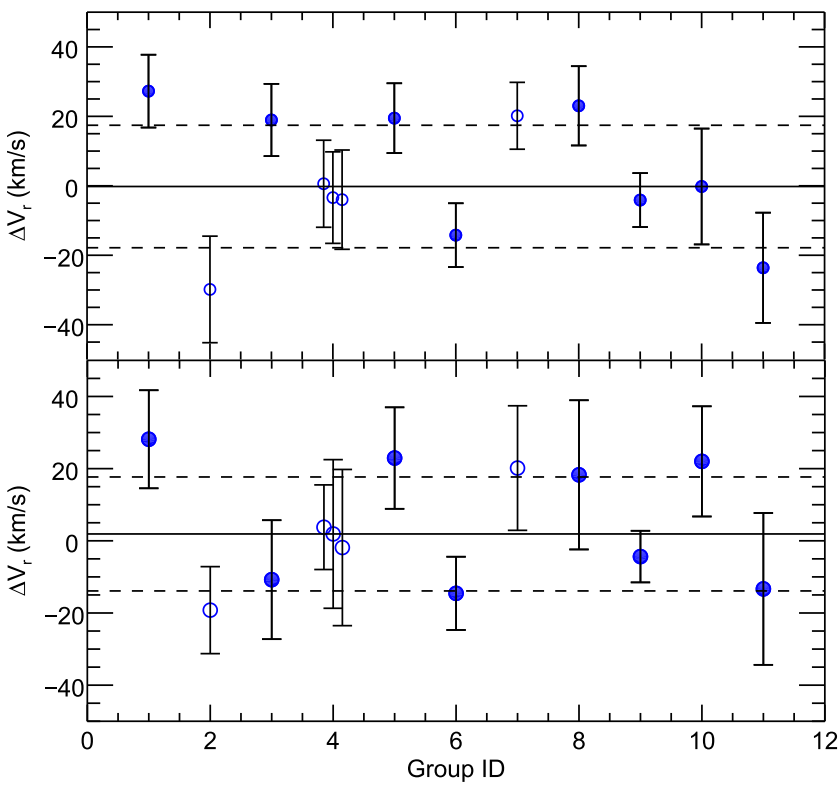

Figure 4. The difference in the radial velocity measurements for the 11 stars that were targeted in more than one mask in P83. The star in Group 4 was observed in three different masks and all difference combinations are plotted. Phx probable members are plotted with solid blue symbols, while non-members are plotted with open symbols. The error bars denote the quadrature combined uncertainties of both measurements. The median of the distribution is indicated with a solid black line and the $\pm 1 \sigma$ level is indicated with dashed lines. Upper panel: velocities are measured after stacking of the individual exposures. Bottom panel: velocities are measured from individual exposures and then the weighted mean values and their uncertainties are calculated.

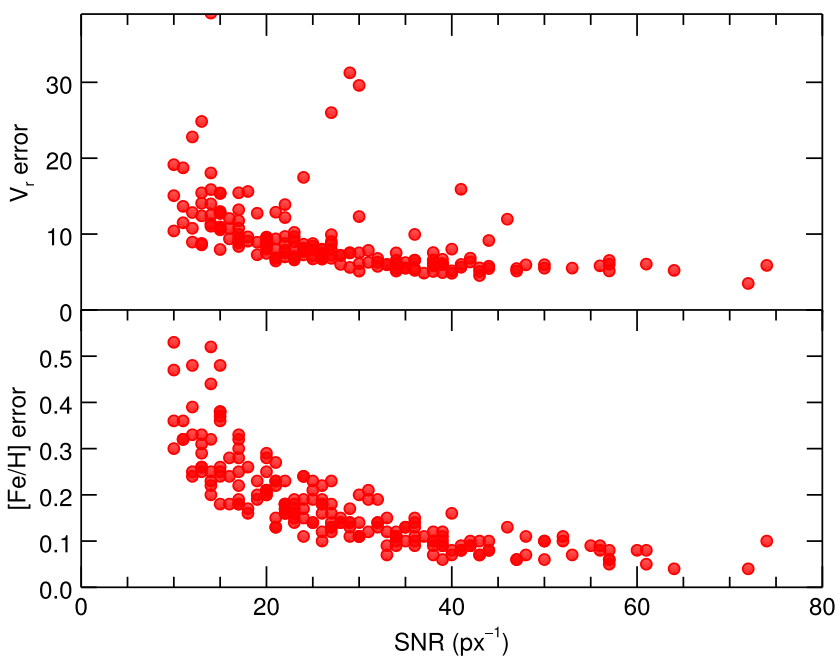

Figure 5. The radial velocity and metallicity uncertainties of the Phx member stars as a function of the SNR in the P83 data set.

deviation of the distribution amounting to $15 \mathrm{~km} \mathrm{~s}^{-1}$ and the median, quadrature combined error for the presented differences being $15 \mathrm{~km} \mathrm{~s}^{-1}$ too. This corroborates our choice of adopting as final velocities those derived as weighted mean of the measurements from individual exposures, rather than from the stacked spectra.

In the upper panel of Fig. 5, we show the final adopted uncertainties of the radial velocities for all probable Phx member stars as function of the SNR of the spectra; the median velocity uncertainty 

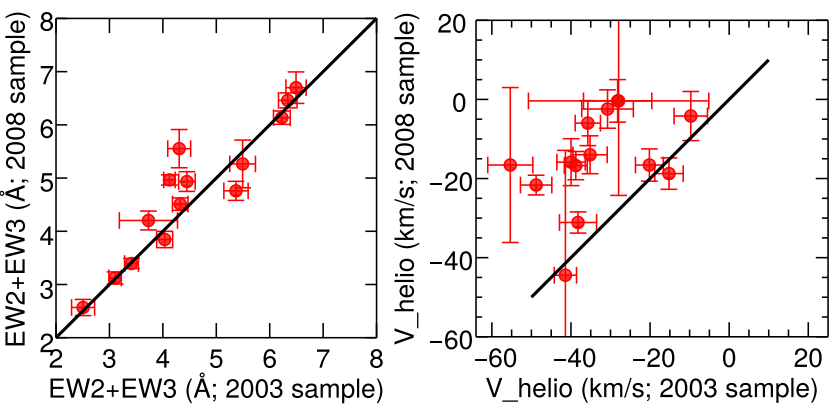

Figure 6. CaT EWs (left-hand panel) and radial velocities (right-hand panel) comparison for the 14 stars in common in the P83 and P71 spectroscopic sets. Black lines indicate equality.

is $8 \mathrm{~km} \mathrm{~s}^{-1}$. The detailed kinematics of the Phx dwarf is presented in Section 5.

\subsection{Comparison of the P71 and P83 data sets}

The radial velocity and CaT EW measurements were performed in the same way for the two data sets. Radial velocities were determined by cross-correlation with the IRAF task fxcor adopting the same synthetic template spectrum and the slit-centring corrections were derived by comparing the centroid of starlight to the centroid of the sky light seen through the slit mask with the grating removed. The corrections for the P71 single MXU setup were between 0.1 and 0.4 pixel after taking into account the fact that the telescope was moved blindly by $0.5 \mathrm{px}$ after the last through-slit image in order to compensate a larger initial offset. The resulting systematic negative offset averages at $-8.6 \mathrm{~km} \mathrm{~s}^{-1}$ with a star-to-star dispersion of $\pm 2.6 \mathrm{~km} \mathrm{~s}^{-1}( \pm 0.1 \mathrm{px})$. The determination of the EWs of the CaT lines is described in Section 3.

Fig. 6 shows the comparison between the velocities and CaT EWs for 14 stars in common in the P71 and P83 spectroscopic samples. While there is a generally good agreement between the measured CaT EWs, there is a clear discrepancy between the velocity measurements, which appears mostly, but not entirely, due to a different velocity zero-point. FORS2 is a low-resolution spectrograph, not optimized for accurate velocity measurements, suffering from flexures. Besides, it was on two different telescopes at the time of the two runs. We could not identify exactly the origin of the velocity difference but it most likely is due to the fore-mentioned blind adjustment of the telescope pointing after the last through-slit image. Thus, we prefer to rely only on the larger and homogeneous P83 sample for the kinematic analysis. On contrary, we deem it safe to combine the two samples for the determination of the CaT EWs, because we measure CaT EWs on the Doppler-corrected spectra. For the stars in common between the P71 and P83 data sets, we adopted the weighted mean values of their CaT EWs.

\section{CAT EW AND METALLICITY CALIBRATION}

The CaT is the most prominent spectral feature in the near-IR spectra of cool stars. The three lines are centred at 8498.02, 8542.09 and $8662.14 \AA$ and their strengths have been extensively used as reliable indicators of the metallicity $([\mathrm{Fe} / \mathrm{H}])$ of individual RGB stars in a large variety of stellar systems: old and relatively metalpoor globular clusters (e.g. Armandroff \& Zinn 1988; Rutledge, Hesser \& Stetson 1997), more metal-rich open star clusters (e.g. Cole et al. 2004; Warren \& Cole 2009; Carrera 2012), but also dwarf galaxies with complex star formation histories and extended metallicity and age spreads (e.g. Armandroff \& Da Costa 1991; Tolstoy et al. 2001; Cole et al. 2005; Koch et al. 2006; Battaglia et al. 2008a; Carrera et al. 2008a,b; Hendricks et al. 2014a). The reliability of the method has been tested via comparison of the CaT $[\mathrm{Fe} / \mathrm{H}]$ with direct $[\mathrm{Fe} / \mathrm{H}]$ measurements from high-resolution spectra of the same stars in systems with composite stellar populations exhibiting a range of metallicities (e.g. dwarf galaxies, the MW bulge; Battaglia et al. 2008a; Vásquez et al. 2015).

A review of the variety of $\mathrm{CaT} \mathrm{EW}-[\mathrm{Fe} / \mathrm{H}]$ calibrations introduced in the literature is beyond the scope of this work and we refer the reader to the various studies for details on the method. Here we use our globular cluster observations to briefly test the Starkenburg et al. (2010) and Carrera et al. (2013) relations, which are calibrated over a large range in $[\mathrm{Fe} / \mathrm{H}](-4<[\mathrm{Fe} / \mathrm{H}]<-0.5$ and $-4<[\mathrm{Fe} / \mathrm{H}]$ $<+0.5$, respectively) that is suitable for our target and take into account the fact that the linearity of the calibration breaks down at $[\mathrm{Fe} / \mathrm{H}] \lesssim-2$ dex.

Both calibrations follow the same functional form:

$[\mathrm{Fe} / \mathrm{H}]=a+b\left(V-V_{\mathrm{HB}}\right)+c \Sigma_{\mathrm{EW}}+d \Sigma_{\mathrm{EW}}^{-1.5}+e \Sigma_{\mathrm{EW}}\left(V-V_{\mathrm{HB}}\right)$,

but assume different coefficients $(a, b, c, d, e)$. The use of the magnitude difference with respect to the horizontal branch $\left(V-V_{\mathrm{HB}}\right)$ was introduced by Armandroff \& Da Costa (1991) in order to compare RGB stars of different luminosities, independently on distance and reddening. A disadvantage, especially in complex stellar populations, is the difficulty to pinpoint a unique HB magnitude for the entire system; however, this effect is expected to introduce an error of $\sim 0.05$ dex, much smaller than the uncertainty intrinsic to the method itself (e.g. Cole et al. 2004, and references therein). We adopted $V_{\mathrm{HB}}=23.9 \mathrm{mag}$ (Holtzman, Smith \& Grillmair 2000) for the Phx dwarf. $\Sigma_{\mathrm{EW}}$ is a linear combination of the EW of the CaT lines: both the Carrera et al. (2013) and the Starkenburg et al. (2010) calibration use an unweighted sum of the EWs of the CaT lines, but while the former uses the three lines, the latter uses only the two strongest lines due to concerns that the weakest line at $8498 \AA$ might introduce more scatter to the calibration (see also Koch et al. 2006; Battaglia et al. 2008a; Hendricks et al. 2014a). It should be noted that also the systemic velocity of the system under consideration may play a role in the choice of the lines to use, as e.g. one of the lines may be more affected by $\mathrm{OH}-$ band sky residuals. Finally, in order to measure the EW of the individual CaT lines, one has the choice between a direct flux integration or fitting a functional form to the line shape to be integrated over a band pass. In the following, we will adopt the prescriptions from Starkenburg et al. (2010) and Carrera et al. (2013) in terms of the band pass over which to integrate the line flux, the linear combination of CaT EWs to be used and obviously the $(a, b, c, d, e)$ coefficients, while experimenting with the method of flux integration (fit or direct flux integration).

We developed our own algorithm to measure the EWs of the CaT lines from the continuum normalized stacked spectra using the MPFIT $^{4}$ package in IDL (Markwardt 2009). We tested EW determination by fitting the CaT lines with pure Gaussians and with true Voigt profiles (a convolution between a Gaussian and a Lorentzian profile) and adopted the $\sigma$-spectra as the flux uncertainty at each wavelength pixel. Additionally, we calculated a simple pixel-topixel integration of the line flux. We note that we trusted the overall continuum normalization of our spectra, which we deem more robust compared to using narrow custom-defined continuum windows

\footnotetext{
${ }^{4}$ http://purl.com/net/mpfit
} 

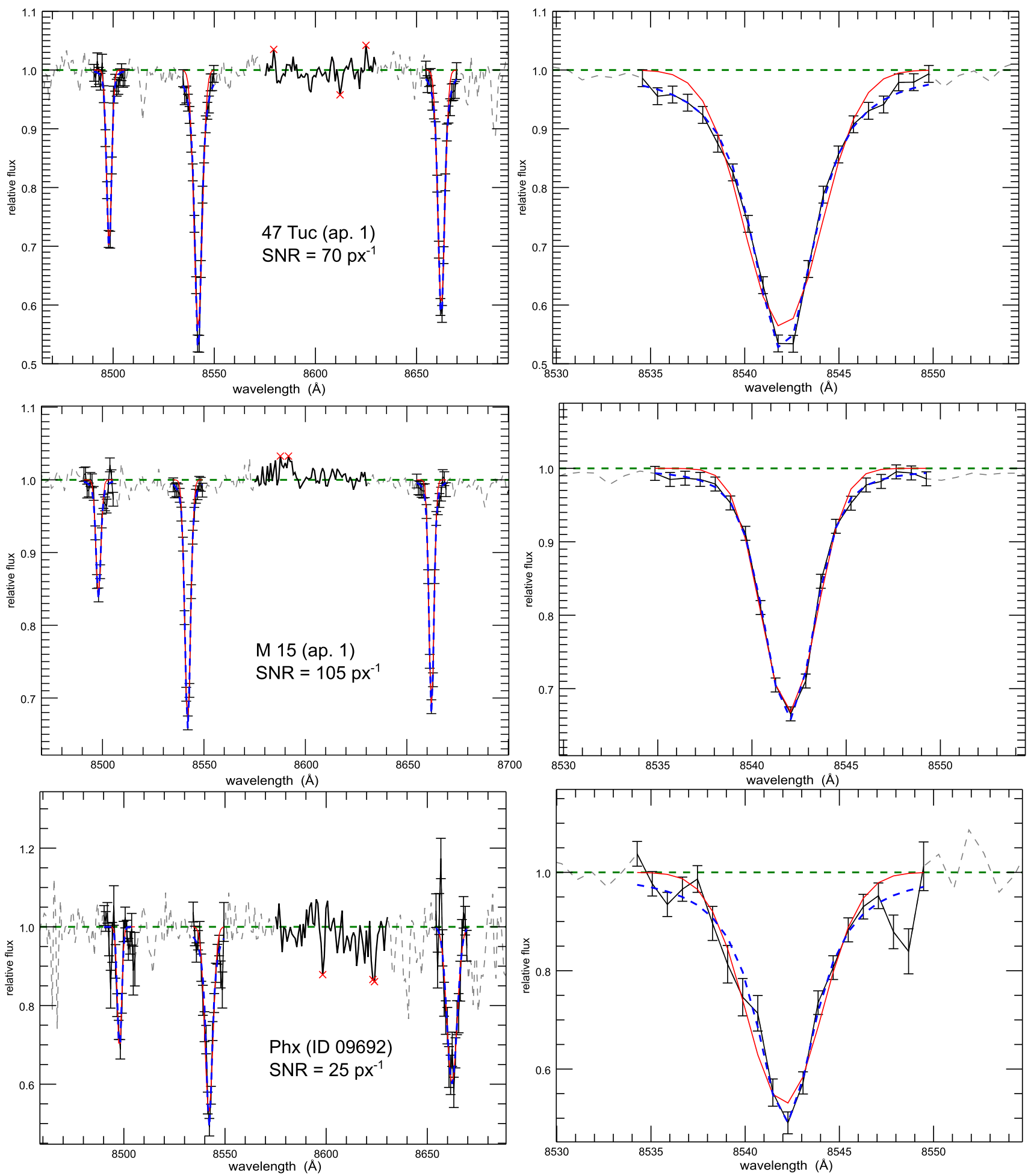

Figure 7. Example spectra of one 47 Tuc (upper row), M 15 (middle row) and Phx (bottom row) star. The left-hand column shows the three Ca lines and the right-hand column shows only the middle 8542 A line. The best-fitting Gaussian profile is indicated with a solid red line and the Voigt profile is indicated with a dashed blue line. The observed spectrum is marked with a dashed grey line. A solid black line with error bars indicates the line band pass region used for the profile fitting and flux integration. One of the defined continuum regions for SNR estimation is marked with a solid black line and the continuum level with a green dashed line. Red crosses indicate continuum points rejected by a $\sigma$-clipping algorithm.

in the calculations, as is also done in e.g. Leaman et al. (2009) with similar data sets.

In Fig. 7, we show example spectra and the fitted model functions for one representative star from the two globular clusters and one star from the Phx galaxy with a typical SNR of 25 per pix. Table 3 summarizes the comparison between the $\mathrm{CaT}[\mathrm{Fe} / \mathrm{H}]$ that we obtain for our calibrating globular clusters 47 Tuc and M 15 when using the two $\mathrm{CaT} \mathrm{EW}-[\mathrm{Fe} / \mathrm{H}]$ calibrations for the various $\mathrm{EW}$ 
Table 3. Median metallicities ([Fe/H]) and measured spreads $\left(\sigma_{[\mathrm{Fe} / \mathrm{H}]}\right)$ for the two calibration globular clusters and the clean Phx sample according to different calibrations and EW measurement methods ('Sum' refers to the direct pixel-to-pixel flux integration).

\begin{tabular}{lccc}
\hline Method & $\begin{array}{c}47 \text { Tuc } \\
-0.72^{a}\end{array}$ & $\begin{array}{c}\text { M 15 } \\
-2.36^{a}\end{array}$ & $\mathrm{Phx}^{b}$ \\
& \multicolumn{4}{c}{ Carrera et al. $(2013)^{c}$} \\
Gauss & $-1.05 \pm 0.10$ & $-2.57 \pm 0.07$ \\
Voigt & $-0.52 \pm 0.13$ & $-2.41 \pm 0.09$ \\
Sum & $-0.42 \pm 0.18$ & $-2.42 \pm 0.16$ \\
\multicolumn{4}{c}{ Carrera et al. $(2013)^{d}$} \\
Gauss & $-1.05 \pm 0.10$ & $-2.57 \pm 0.07$ \\
Voigt & $-0.67 \pm 0.11$ & $-2.33 \pm 0.08$ \\
Sum & $-0.71 \pm 0.12$ & $-2.46 \pm 0.10$ \\
& \multicolumn{4}{c}{ Starkenburg et al. $(2010)$} \\
Gauss & $-0.90 \pm 0.09$ & $-2.37 \pm 0.08$ & $-1.67 \pm 0.62$ \\
Voigt & $-0.71 \pm 0.09$ & $-2.27 \pm 0.10$ & $-1.53 \pm 0.59$ \\
Sum & $-0.68 \pm 0.09$ & $-2.26 \pm 0.11$ & $-1.50 \pm 0.62$
\end{tabular}

${ }^{a}[\mathrm{Fe} / \mathrm{H}]$ according to the catalogue of Harris (1996, 2010 edition).

${ }^{b}$ Only probable Phx member stars are included.

${ }^{c}$ Using the Cenarro et al. (2001) band windows as in Carrera et al. (2013).

${ }^{d}$ Using the Armandroff \& Zinn (1988) band windows.

measurement methods (pixel-to-pixel integration, Gaussian and Voigt fits). In line with other studies in the literature, it can be clearly seen that the Gaussian fit is a poor representation of the line shape at high metallicity, missing the wings of the line, which then leads to underestimating the $[\mathrm{Fe} / \mathrm{H}]$ abundance of 47 Tuc for both CaT calibrations; on the other hand, the Voigt fits not only offer a suitable representation of the CaT line profiles, but are also less prone to subtle fluctuations of the flux due to the presence of weak lines and noise that affects more the direct flux integration; hence we adopt the EWs inferred from Voigt fits for the rest of the discussion in this paper.

The Carrera et al. (2013) calibration is based on observations from multiple instruments including FORS2 but with higher spectral resolution of $\sim 5000$. They adopted the line band windows of Cenarro et al. (2001), which are broad $(29,40,40 \AA$ for the three lines, respectively) and account for the extended line wings at high metallicity. In our analysis, such broad-band passes turn out to be, however, very sensitive to small uncertainties of the continuum zero-point. Our Voigt profile fit procedure predicts artificially large Lorentzian broadening for the metal-rich stars of 47 Tuc, which leads to very large EWs and hence higher than expected metallicity. At the metal-poor regime, the calibration reproduces well the metallicity of M 15 (see Table 3). We note that we can reproduce the expected metallicities of the two globular clusters with the Carrera et al. (2013) calibration if we use the narrower line band passes proposed by Armandroff \& Zinn (1988, 16, 20, 18 Å, respectively).

The Starkenburg et al. (2010) relation, on the other hand, was not calibrated up to metallicities as high as in the Carrera et al. (2013) study and uses band windows of $15 \AA$ for the two stronger CaT lines following the approach described by Battaglia et al. (2008a). The advantage of such narrow integration windows is that the calibration is not sensitive to small continuum uncertainties and effects such as random noise or weak spectral lines in the vicinity of the CaT. It might, however, be less sensitive at high metallicities due to the exclusion of the extended $\mathrm{Ca}$ II line wings from the EW integration. Nevertheless, using this calibration, we reproduced very well the metallicities of both 47 Tuc at the high metallicity end and M 15 in the low-metallicity regime. Therefore, we decided to adopt

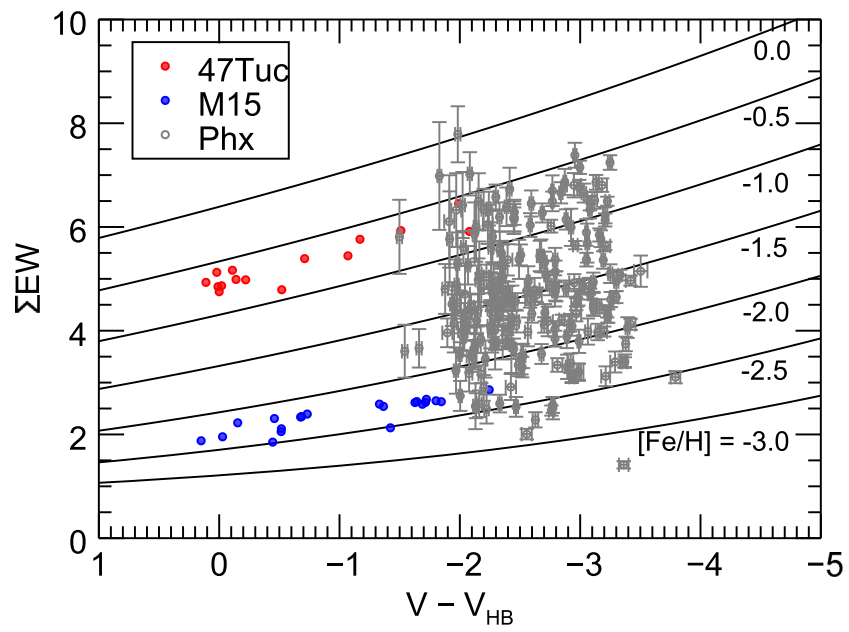

Figure 8. The sum of the EWs of the two stronger CaT lines as a function of $V-V_{\mathrm{HB}}$ for the clean Phx sample and the globular cluster stars. Lines of equal metallicity according to the Starkenburg et al. (2010) calibration are overplotted with solid black lines.

Starkenburg et al. (2010) calibration to derive the metallicities of the Phx stars, based on the EWs from the Voigt fits integrated over a band pass of $15 \AA$. The Starkenburg et al. (2010) calibration was obtained using synthetic spectra of resolution $R=6500$. This is larger than our FORS2 resolution but we confirmed by examining synthetic spectra with the average parameters of the 47 Tuc stars, convolved to different instrument resolutions, that the signal within the $15 \AA$ band windows is practically the same.

We used two approaches to compute the uncertainties of the EW measurements for the observed stars. The MPFIT code provides formal $1 \sigma$ errors based on the covariance matrix of the fitting parameters. Since our line profile fits were weighted with the corresponding $\sigma$-spectra, the errors calculated in this way should be representative of the true uncertainties. As a second approach to compute the uncertainties, we used the empiric formula proposed by Cayrel (1988) in the form from Hendricks et al. (2014a):

$\Delta_{\mathrm{EW}}=1.725 \mathrm{SNR} \sqrt{\sigma_{\text {Gauss }}}$,

where $\sigma_{\text {Gauss }}$ is the $1 \sigma$ broadening of the Gaussian profile fit, which we assumed to be very close to the equivalent value of the Voigt profile fit. The Cayrel formula is based solely on the resolution and the SNR of the spectra. We find the uncertainties inferred from the two methods to be strongly correlated but the errors based on the goodness of the fit are systematically larger for both the good-quality globular cluster spectra and the fainter Phx spectra. We adopt the latter as the final uncertainties on the EW measurements.

The errors on the EWs of the two strongest CaT lines were combined in quadrature and we used the bottom and upper EW boundaries to calculate the uncertainties on the metallicity estimates. In the bottom panel of Fig. 5, we show the final $[\mathrm{Fe} / \mathrm{H}]$ uncertainties for all probable Phx member stars, with a median uncertainty of 0.16 dex.

In Fig. 8, we plot $\Sigma_{\mathrm{EW}}$ as a function of the $V$ magnitude above the HB. One can see the accurate metallicity estimates for the two calibration globular clusters $(-0.72$ and -2.36 dex for 47 Tuc and M 15, respectively, according to the catalogue of Harris 1996, 2010 edition) and their small metallicity spread. On the other hand, a large metallicity spread is already evident for the Phx dwarf galaxy. The metallicity gradients and chemical enrichment of $\mathrm{Phx}$ is further discussed in Section 6. 

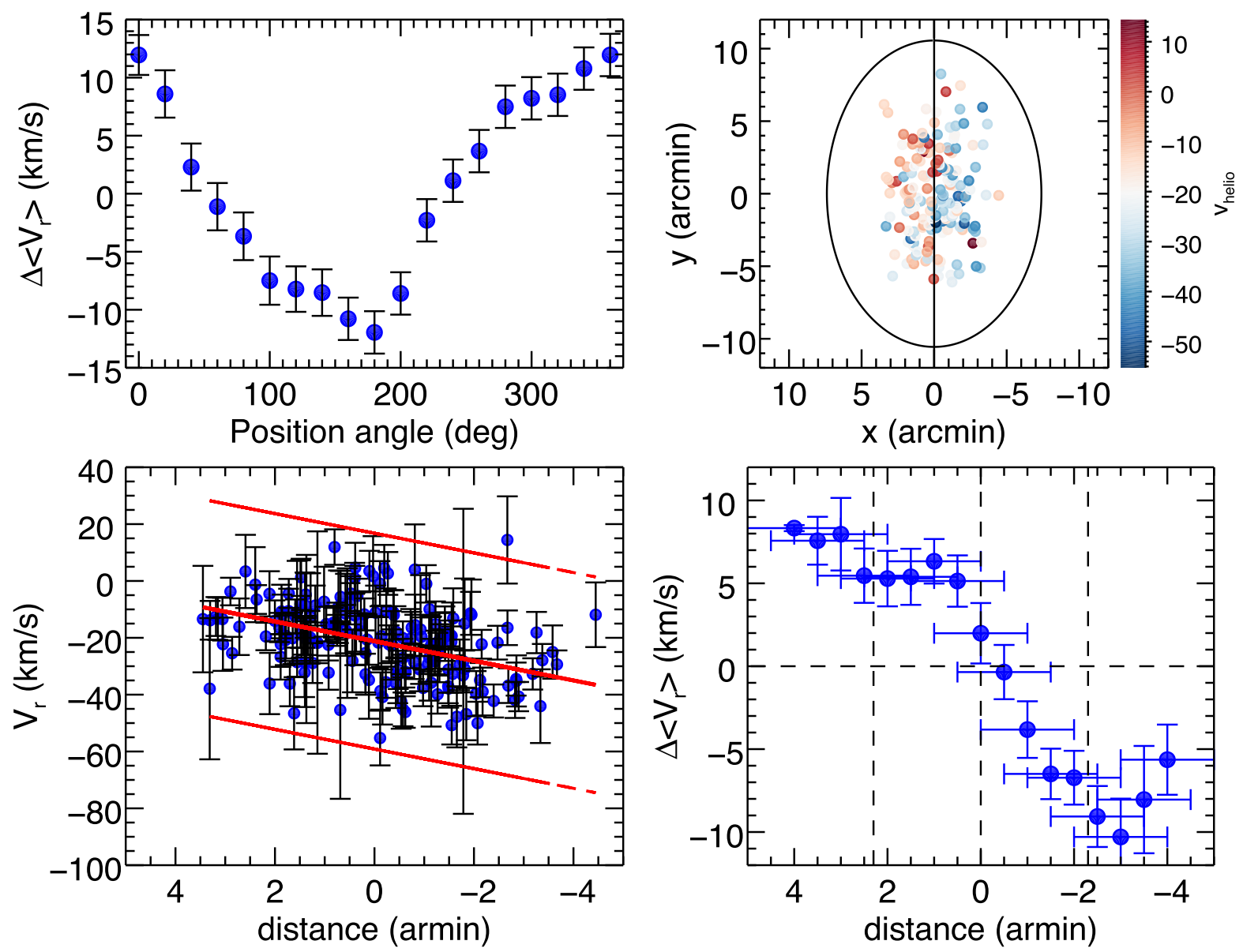

Figure 9. Upper left panel: difference between the mean velocities on each side of the galaxy with respect to a line passing through its centre at a given position angle. Upper right panel: spatial location of the observed Phx stars in a coordinate system where the axes are rotated to be aligned with the galaxy's major and minor axes. The colour code denotes the line-of-sight velocity. The tidal ellipse (Battaglia et al. 2012) and the adopted rotation axis are superimposed. Bottom left panel: velocity gradient - the radial velocity of each probable member star with respect to a spatial axis perpendicular to the rotation axis of the galaxy. The red lines correspond to an error-weighted linear fit to the data \pm 3 times the intrinsic velocity dispersion. Bottom right panel: rotation profile of Phx, where the abscissa shows the distance from the centre of the galaxy along the axis perpendicular to the rotation axis and the ordinate shows the mean offset from its systemic velocity in different overlapping bins. The horizontal error bars indicate the size of the selected bins, while the vertical error bars indicate the formal uncertainty of the mean velocity offset. The vertical dashed lines indicate the half-light radius of Phx.

\section{SELECTION OF Phx MEMBERS}

With Galactic coordinates $l \sim 270^{\circ}, b \sim-79^{\circ}$, Phx is located in a region of the sky that is not heavily contaminated by foreground stars. On the other hand, its systemic radial velocity is not well separated from the line-of-sight velocity distribution of the MW disc.

The first pass of our Phx member selection was made on the basis of the stars' magnitudes and colours, retaining targets whose location on the CMD is consistent with RGB stars at the distance of the Phx dwarf galaxy (see Fig. 2); this excluded 26 objects.

Since the radial velocities for the P71 FORS2 sample could not be reliably combined with the P83 sample, the membership of these stars was further assessed solely on their location in the $\mathrm{FoV},[\mathrm{Fe} / \mathrm{H}]$ distribution and the strength of the $\mathrm{Mg}$ I line at $8807 \AA$, which is a proxy for surface gravity (Battaglia \& Starkenburg 2012). Since this sample covers the innermost region of $\mathrm{Phx}$, its metallicity distribution follows very well the metallicity distribution measured from the P83 sample, and the EWs of the Mg I line are consistent with all stars being red giants, showing a smooth correlation with the EWs of the CaT lines with no obvious outliers, we consider that all these stars are Phx members. In further support of this conclusion, stars of this sub-sample do not show a large velocity dispersion.

For the P83 sample, we excluded stars that have obviously outlying radial velocities; then we applied further radial velocity cuts by inspecting the velocity gradient plot (see the bottom left panel of Fig. 9) until only stars within three times the observed (error inflated) velocity dispersion were left. The second step was repeated iteratively several times until convergence. The measurement of the $\mathrm{EW}$ of the Mg I line was possible for 135 likely member stars. Its strength is consistent with all these stars being red giants within the measurement uncertainty according to the selection criteria proposed by Battaglia \& Starkenburg (2012).

The sample of probable member stars so derived consists of 178 objects with both line-of-sight velocity and $[\mathrm{Fe} / \mathrm{H}]$ measurements and further 18 objects with $[\mathrm{Fe} / \mathrm{H}]$ measurements (for a total of 196 members)

To assess the remaining contamination of the sample, we retrieved a Besançon simulation of MW stars (Robin et al. 2003) in the observed FoV that have $V$ magnitude between 20.0 and 22.5 and colour $0.8<V-I<2.2$. The Galactic model predicts $\sim 10$ stars that would pass all our selection criteria. This number can be regarded as 
an upper limit because the surface number density of Phx is larger than the surface density of MW contaminants at most radii, making it more likely to pick a Phx member star when placing slits on to targets.

In Kacharov et al. (in preparation), we also determine membership probability on the basis of the observed spatial distribution of Phx stars as well as the kinematic properties predicted by axisymmetric Jeans mass modelling of the system, in presence of foreground contamination. This approach predicts 191 stars with a probability of membership larger than 0.8 , in very good agreement with the selection made by hard-cuts described above.

We have verified that the Phx properties presented in this paper are unmodified by the choice of one or the other approach for membership selection; the only change worth of notice is that the three most external stars would not be classified as members in the Jeans modelling. In the following, we adopt the selection made on the hard-cuts just for the sake of making the analysis independent on the details of the modelling.

\section{KINEMATIC PROPERTIES}

We used a maximum likelihood approach (van de Ven et al. 2006; Walker et al. 2006) to compute the systemic heliocentric radial velocity of the Phx dwarf galaxy $\left(V_{\text {helio }}=-21.2 \pm 1.0 \mathrm{~km} \mathrm{~s}^{-1}\right)$ and its intrinsic velocity dispersion $\left(9.3 \pm 0.7 \mathrm{~km} \mathrm{~s}^{-1}\right.$, after correcting for the velocity gradient) taking into account the individual uncertainties of all the stars in the clean sample of probable member stars. The corresponding systemic velocity in the Galactic standard of rest is $V_{\mathrm{GSR}}=-108.6 \pm 1.0 \mathrm{~km} \mathrm{~s}^{-1}$, 5 i.e. $\mathrm{Phx}$ is approaching the MW. At a distance of $\sim 450 \mathrm{kpc}$, however, it has likely not yet encountered the hot Galactic halo extending to about $250-300 \mathrm{kpc}$ and is moving through the Local Group medium, where ram pressure may be responsible for stripping of the remaining $\mathrm{H}$ I gas. Its velocity with respect to the Local Group centre of mass is $-100.8 \mathrm{~km} \mathrm{~s}^{-1}$.

The systemic velocity is consistent with the determination of -13 $\pm 9 \mathrm{~km} \mathrm{~s}^{-1}$ obtained by Irwin \& Tolstoy (2002) from a much smaller sample of seven stars. Worth noting is the excellent agreement of our measurements with the velocity and velocity dispersion of the $\mathrm{H}$ i cloud $\left(V_{\mathrm{HI}}=-23 \mathrm{~km} \mathrm{~s}^{-1}, \sigma_{\mathrm{HI}}=6 \mathrm{~km} \mathrm{~s}^{-1}\right)$ proposed by St-Germain et al. (1999) as physically associated with Phx. The $-23 \mathrm{~km} \mathrm{~s}^{-1}$ cloud is more compact than other Galactic gas clouds along the line of sight (St-Germain et al. 1999) and although not centred on Phx, its location and velocity structure are consistent with currently being stripped from the dwarf galaxy via ram pressure or SNe feedback from the last episode of star formation (Gallart et al. 2001). However, we caution that at such low velocity, the possibility of MW gas contamination is still high.

It is well known that dwarf galaxies inhabiting dense environments such as within the virial radii of the MW or M31 are devoid

\footnotetext{
${ }^{5}$ This is determined using the formula in Binney \& Tremaine (1987), with an LSR velocity $v_{\mathrm{LSR}}=220 \mathrm{~km} \mathrm{~s}^{-1}$ at the distance of the Sun from the MW centre $\left(\mathrm{R}_{\odot}=8 \mathrm{kpc}\right)$ and a solar motion of $(U, V, W)=(10$, $5.25,7.17) \mathrm{km} \mathrm{s}^{-1}$ (Dehnen \& Binney 1998), where $U$ is radially inwards, $V$ positive in the direction of Galactic rotation and $W$ towards the north Galactic pole. If we were to use $8.3 \mathrm{kpc}$ from distance of the Sun from the Galactic Centre (Gwinn, Moran \& Reid 1992), a local rotation speed of $236 \mathrm{~km} \mathrm{~s}^{-1}$ (Bovy, Hogg \& Rix 2009) and a solar motion $(U, V, W)=(11.1$, $12.24,7.25) \mathrm{km} \mathrm{s}^{-1}$ (Schönrich, Binney \& Dehnen 2010), $v_{\mathrm{GSR}}$ would be $-114.2 \mathrm{~km} \mathrm{~s}^{-1}$ instead of $-108.6 \mathrm{~km} \mathrm{~s}^{-1}$.

${ }^{6}$ The velocity with respect to the Local Group centre of mass is determined using the formula provided in Tully et al. (2008).
}

of gas, except for relatively massive systems such as e.g. the Magellanic Clouds or IC 10. The fact that such a small system as Phx has been able to form stars until almost present day (Hidalgo et al. 2009) and is likely to still contain gas, together with its large distance and approaching velocity, suggests that Phx is likely to enter the MW virial radius for the first time and has evolved undisturbed from interactions with large galaxies. However, assuming a purely radial orbit and $10^{12} \mathrm{M}_{\odot}$ point mass for the MW, we estimate an orbital period of the order of $10 \mathrm{Gyr}$ and an apocentric distance of $\sim 1 \mathrm{Mpc}$. Even on such an extended orbit, Phx has had time for at least one pericentric passage, hence velocity and distance alone cannot rule out the possibility of a close encounter with the MW in the past.

In this section, we make a detailed kinematic analysis of $\mathrm{Phx}$ using the same maximum likelihood technique to compute mean velocities and intrinsic dispersions for selected parts of the stellar sample. Although we use the heliocentric radial velocities in the rest of the paper, we confirmed that due to the small angular size of Phx, there is only a constant difference between the heliocentric and GSR velocities for all stars in the field with a standard deviation of just $0.15 \mathrm{~km} \mathrm{~s}^{-1}$, which is too small to be accounted for in our sample.

\subsection{Rotation}

We followed the procedure by Walker et al. (2006) to detect velocity gradients by measuring the difference between the mean velocities on either side of a line passing through the galaxy's centre and rotated at different position angles (Fig. 9; upper left panel). Although the resulting curve is not symmetric, there is a clear maximum at $0^{\circ}$ (minimum at $180^{\circ}$ ) corresponding to a strong prolate rotation ( $V_{\text {rot }} \sin i=5.5 \pm 0.6 \mathrm{~km} \mathrm{~s}^{-1}$, where $i$ is the unknown inclination angle). The velocity gradient can be clearly appreciated by eye as well, e.g. on a plot showing the radial velocities across the FoV of Phx (Fig. 9; upper right panel).

To further test the significance of the rotation detection, we performed a Monte Carlo test by simulating a mock galaxy that follows a 2D Sérsic distribution with ellipticity of 0.3 and contains $10^{4}$ stars. This mock galaxy has a Gaussian velocity distribution with an intrinsic dispersion of $9 \mathrm{~km} \mathrm{~s}^{-1}$ and no intrinsic rotation. We extracted the simulated stars that are closest to the observed $x, y$ positions in Phx and further reshuffled their mock velocities according to the velocity uncertainties measured in the observed sample at each given position. Then we proceeded measuring the average rotation and rotation axis position angle of the resulting mock sample. This was repeated 500 times. Fig. 10 shows the estimated position angle versus the average rotation amplitude of all Monte Carlo realizations. Rotation axis of $0^{\circ}$ corresponds to a prolate rotator and $\pm 90^{\circ}$ corresponds to an oblate rotator. Indeed, it is much more likely to detect a false prolate rotator than an oblate one. Our detection of prolate rotation in $\mathrm{Phx}$, however, is at $\gtrsim 5 \sigma$ significance level.

In the bottom left panel of Fig. 9, we plot the radial velocities of the $\mathrm{Phx}$ probable member stars projected on to the axis of maximum velocity gradient (i.e. the axis perpendicular to the rotation axis). An error-weighted linear fit is overplotted. That corresponds to a simple rigid body rotation model. However, to better explore the rotation pattern of $\mathrm{Phx}$, we construct a rotation profile in the bottom right panel of Fig. 9 by plotting the difference between the systemic radial velocity of the galaxy and the mean velocity inferred from the stars in multiple overlapping bins along the axis of maximum velocity gradient. We see that the mean velocity rises smoothly from the inner parts of this galaxy, starting to flatten out around $V_{\max } \sin i \sim 8 \mathrm{~km} \mathrm{~s}^{-1}$ beyond the half-light radius $\left(r_{\mathrm{h}}=2.3 \mathrm{arcmin}\right.$; 


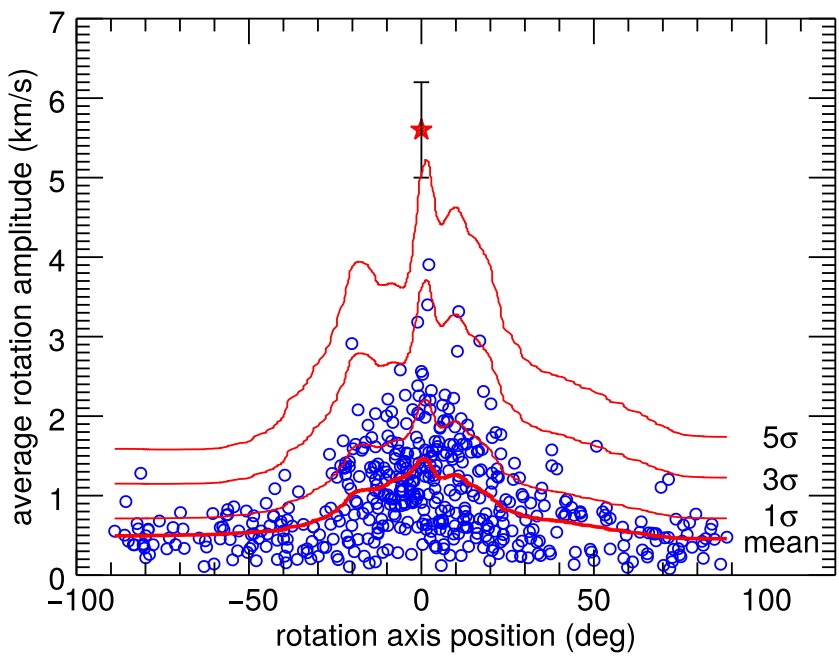

Figure 10. Monte Carlo realization of false rotation detections. The plot shows the detected false rotation signal as a function of the rotation axis position $\left(0^{\circ}\right.$ - prolate system, $\pm 90^{\circ}$ - oblate system $)$. The running mean is overplotted with a thick red line and the $1 \sigma, 3 \sigma, 5 \sigma$ significance levels are overplotted with thin red lines. The detection in Phx is indicated by a red star.

Battaglia et al. 2012). Even though the exact shape cannot be pinned down with this data set, it is evident that Phx displays a velocity gradient that is aligned with its projected minor axis.

Velocity gradients detected in objects with a large angular extent, such as MW satellites, can arise due to the projection of the $3 \mathrm{D}$ systemic motion of the galaxy on to the line of sight towards the individual stars; such gradients would mimic solid body rotation. We explore what tangential motion could give rise to the velocity gradient we observe for Phx. Following the formalism by Strigari, Frenk \& White (2010), let us consider a Cartesian reference frame with the $z$-axis pointing towards the observer, the $x$-axis positive in the direction of decreasing right ascension and the $y$-axis in the direction of increasing declination. In the small angle approximation, the $3 \mathrm{D}$ systemic motion of the galaxy projects on to the line of sight of each star as

$u=v_{x} \times(x / D)+v_{y} \times(y / D)-v_{z} \times\left(1-\left(R^{2} / D^{2}\right) / 2\right)$,

where $R=\sqrt{x^{2}+y^{2}}, D$ is the distance to the galaxy and $v_{x}, v_{y}$ and $v_{z}$ are the galaxy's systemic velocity components. In the case of $\mathrm{Phx}$, we trace the velocity gradient out to $R \sim 4 \operatorname{arcmin}$ approximately along the right ascension axis; the term $R^{2} / D^{2}$ and $v_{y}$ can be therefore neglected and the above equation reduces to $u=v_{x} \times(x / D)$. If we take the mean velocity $u$ at $x \sim 4 \operatorname{arcmin}$ to deviate $\sim 5-8 \mathrm{~km} \mathrm{~s}^{-1}$ from the systemic heliocentric velocity $v_{z}$, then this would correspond to a tangential motion $v_{x}$ between 4000 and $6600 \mathrm{~km} \mathrm{~s}^{-1}$ ! Such large values are hardly reconcilable with the velocity dispersion of the Local Group, as well as with expectations for sub-haloes found within $\sim 1 \mathrm{Mpc}$ from MW-sized haloes in the Aquarius simulations as well as Via Lactea-II and GHALO, be them bound or unbound to the main halo (e.g. see analysis of Boylan-Kolchin et al. 2013). Hence, the detected velocity gradient is not to be attributed to perspective effects, which could account to about $1 / 10$ of the detected amplitude.

We conclude that the most likely possibility is that $\mathrm{Phx}$ displays prolate rotation, with a rotation-to-dispersion ratio approaching 1 in the outer parts. The clear signal of prolate rotation in $\mathrm{Phx}$ is puzzling from a dynamical point of view. Prolate rotation has been occasionally observed in giant elliptical galaxies, where the morphology is determined by the shape of a triaxial dark matter halo (e.g. Schechter \& Gunn 1978; Emsellem et al. 2004; van den Bosch et al. 2008), while most dSphs have been found to be oblate. An interesting case is the Andromeda II dSph, which, to our knowledge, is the only other example of a prolate rotating dwarf galaxy (Ho et al. 2012). Amorisco et al. (2014) detected a cold stellar stream in the field of this galaxy and suggested that a merger event has significantly altered its kinematics. Fouquet et al. (2017) provide further evidence from $\mathrm{N}$-body and hydrodynamical simulations that a major merger was responsible for the prolate rotation in And II. It is possible that Phx evolution mostly unaffected by interactions with the MW may have allowed it to experience a major merger with another dwarf - something extremely unlikely in the vicinity of a giant galaxy (Tremaine 1981; De Rijcke et al. 2004).

It is intriguing to note that the spatial distribution of young stars in $\mathrm{Phx}$ is clearly tilted by $\sim 90^{\circ}$ with respect to $\mathrm{Phx}$ older stellar component (Martínez-Delgado, Gallart \& Aparicio 1999b; Hidalgo et al. 2009; Battaglia et al. 2012), i.e. it is aligned with the projected minor axis of the main body and the direction of the maximum velocity gradient. It is possible that the events that caused the prolate rotation are also responsible for the peculiar distribution of the recently formed stars.

An alternative possibility for the appearance of this odd velocity gradient may be sought if Phx did experience one close encounter with the MW and displays clues of tidal deformation.

In the upcoming paper (Kacharov et al., in preparation), we will examine both the rotation signal and the velocity dispersion profile via orbit modelling and Jeans modelling of Phx as a prolate, axisymmetric body. We defer Phx mass estimates, as well as the presentation of the velocity dispersion profile to that paper.

\section{METALLICITY PROPERTIES}

We find that $\mathrm{Phx}$ is a relatively metal-poor system with a large intrinsic metallicity spread: i.e. by approximating the metallicity distribution function (MDF) with a Gaussian, the maximum likelihood value for the mean metallicity of the observed sample is $[\mathrm{Fe} / \mathrm{H}]=-1.49 \pm 0.04 \mathrm{dex}$ with an intrinsic dispersion $\sigma_{[\mathrm{Fe} / \mathrm{H}]}=0.51 \pm 0.04 \mathrm{dex}$.

\subsection{Spatial variations}

In the upper left panel of Fig. 11, we show the metallicity of each probable member star as a function of elliptical radial distance from the galaxy's centre. There is a clear metallicity gradient with more metal-rich stars located in the central regions and more metal-poor stars spread out at all radii. An error-weighted linear fit suggests a central metallicity of $-1.14 \pm 0.02$ dex, which gradually decreases by $0.13 \pm 0.01 \mathrm{dex} \operatorname{arcmin}^{-1}\left(0.23 \mathrm{dex}\right.$ per $\left.r_{c}\right)$; the trend is confirmed by a running average. The upper right panel of Fig. 11 shows the 2D metallicity distribution of our sample of Phx member stars. It seems that the most metal-rich giant stars (representative of the intermediate-age population, see Section 6.2) are aligned with the central bar-like feature of young main-sequence stars (see MartínezDelgado et al. 1999b; Hidalgo et al. 2009; Battaglia et al. 2012) and indicated by the position of the small, inner ellipse in the figure. This radial metallicity gradient is fully in agreement with the radial star formation history (SFH) gradient discussed by Hidalgo et al. (2009), according to which young- and intermediate-age stars, which are the most metal-rich ones, are concentrated in the central part, gradually disappearing at large galactocentric radii. Thus, the origin of the 

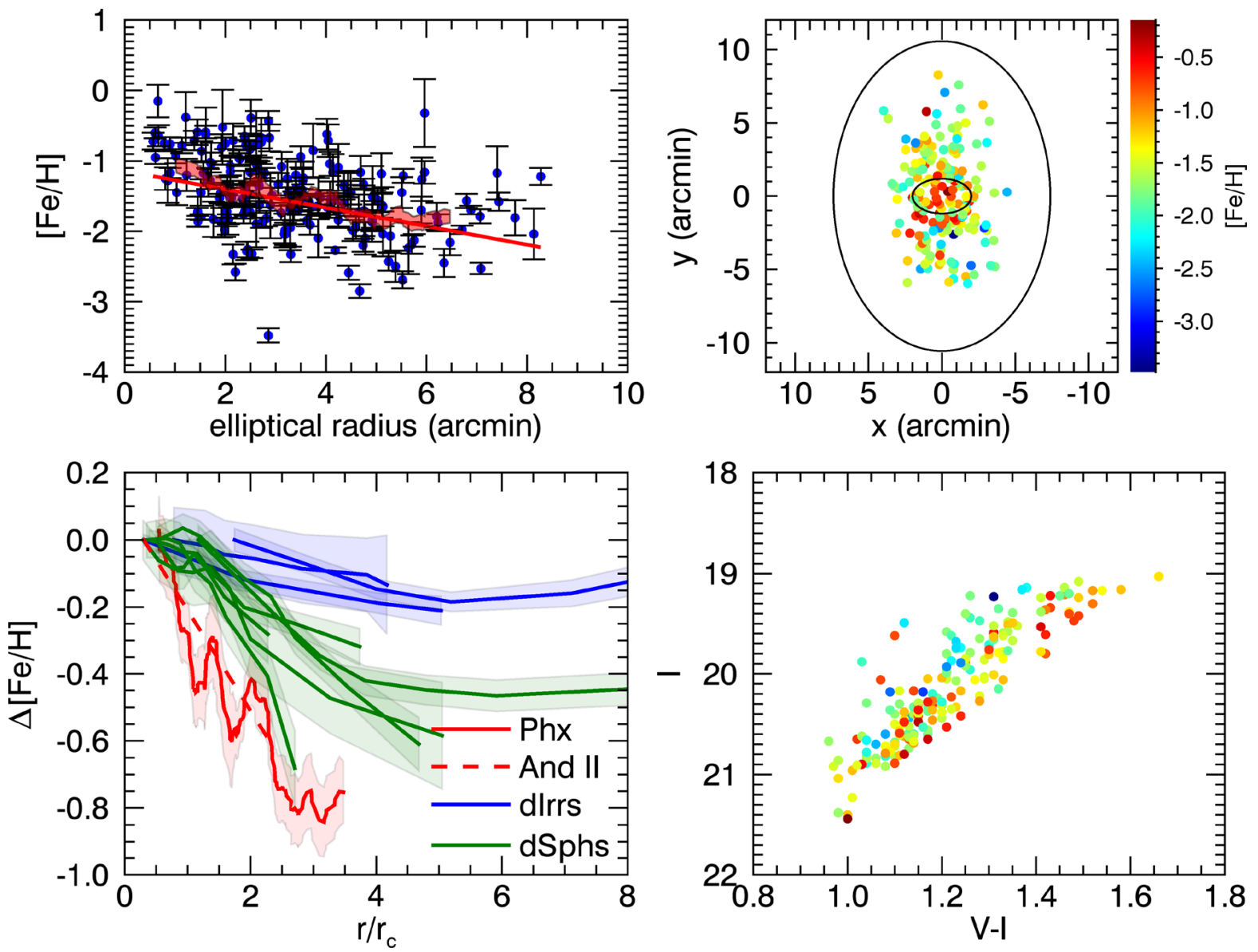

Figure 11. Upper left panel: metallicity gradient in the Phx galaxy - the metallicity of each star as a function of its elliptical radius is shown by the circles with error bars. An error-weighted linear fit to the data is indicated with a red line and an error-weighted, running mean computed at the position of each star based on the 20 stars with closest elliptical radial coordinates is indicated with a transparent, red curve. The thickness of the curve corresponds to the uncertainty of the mean. Upper right panel: spatial extent of the observed Phx probable member stars. The colour code denotes the metallicity. The outermost and innermost ellipse indicate the nominal King tidal radius and the approximate location of the young stars, respectively (Battaglia et al. 2012). Bottom left panel: the metallicity gradient of Phx (red) is compared to those of MW dSphs (green) and gas-rich objects (WLM, SMC, LMC, in blue) as presented in (Leaman et al. 2013). The metallicity gradient of And II (red dashed line Ho et al. 2015) is also presented assuming a core radius of 5.2 arcmin (McConnachie \& Irwin 2006). Bottom right panel: location of Phx member stars on the CMD: a clear colour-metallicity correlation is visible.

metallicity gradient is actually to be found in the age gradient present in the galaxy and in the existence of an age-metallicity relation. The gradient would be much flatter if only older stars were considered.

Negative metallicity gradients of varying steepness have been observed in most MW and in some M $31 \mathrm{dSphs}$ of comparable luminosity to Phx. It is remarkable that the only other Local Group dSph with prolate rotation discovered so far, And II, has a metallicity gradient of similar steepness as Phx and both are the systems in which the steepest gradient has been measured. On the contrary, there are indications that shallower gradients are present in more massive systems (Fig. 11, bottom left, see also fig. 10 in Ho et al. 2015). The fact that such steep metallicity gradients are present in low-mass dwarf galaxies inhabiting a dense environment (i.e. satellites of the MW or M 31) and in a system found beyond the Galactic virial radius and possibly at its first approach towards the Galaxy might point to metallicity gradients being an intrinsic property of these small galaxies, possibly linked to the age gradients generally found in them (and very pronounced, particularly, in Phx and And II). The result appears slightly more difficult to reconcile with the hypothesis that a higher rotational-versus-pressure support produces shallower metallicity gradients (Leaman et al. 2013), given the larger average rotation of Phx (and And II) with respect to classical MW dSphs. If, however, the prolate rotation in these systems was caused by an accretion or merger event, it is not excluded that Phx or And II rotation might have been lower in the past, bringing them in better agreement with this hypothesis.

The steep metallicity gradient of Phx is unlikely to be an artefact, as metallicities were determined using the same spectral lines and calibration as for most of the objects analysed in Leaman et al. (2013). Since Phx was able to form stars until very recently, while star formation in the majority of MW dSphs stopped long ago ( $7-8$ Gyr ago), it is reasonable to speculate that the $\mathrm{H}$ I gas had time to concentrate further in the centre of $\mathrm{Phx}$ (provided angular momentum was not acting as a 'centrifugal barrier'; see Schroyen et al. 2011). From the age-metallicity relation derived in Section 6.2, it is evident that the more metal-rich stars, which are found within the inner 3 arcmin, are the youngest among the targeted RGB stars. If star formation in $\mathrm{Phx}$ would have stopped a few gigayears ago, it is very likely that we would have observed a shallower gradient.

In the bottom right panel of Fig. 11, we show the location of Phx members on the CMD. A colour-metallicity dependence is clearly detectable, although slightly smeared due to the age-metallicity 


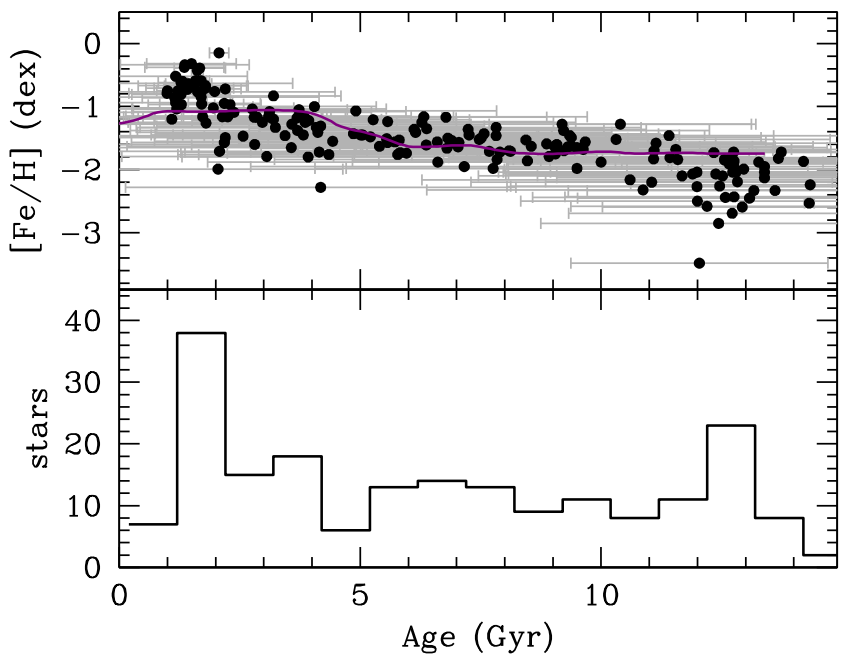

Figure 12. Age-metallicity relation for Phx stars in our VLT/FORS2 MXU sample, derived using equation (4) (top). The bottom panel shows the age distribution. The purple solid line indicates the age-metallicity relation derived by (Hidalgo et al. 2009) from HST CMD diagrams.

degeneracy. The metal-poor stars are found predominantly at bluer colours, while the more metal-rich stars are found at redder colours.

\subsection{Age-metallicity relation}

The spatially resolved star formation and chemical evolution history of Phx were obtained by Hidalgo et al. (2009) from deep Hubble Space Telescope (HST)/WFPC2 CMDs reaching out to $\sim 5$ arcmin from the centre. The age-metallicity relation shows the metallicity to be approximately constant around $[\mathrm{M} / \mathrm{H}]=-1.7$ in the first $7 \mathrm{Gyr}$ of evolution, to then increase more quickly to $[\mathrm{M} / \mathrm{H}] \sim-1.1$ and finally flatten around that value or even decrease (Fig. 12).

Deriving stellar ages for RGB stars is hampered by the wellknown age-metallicity degeneracy and carries large uncertainties. However, we can attempt to estimate relative stellar ages of our sample of RGB stars since we have obtained spectroscopic metallicities from the strength of the CaT lines, hence independently from the CMD information.

Here we use the relation by Carrera et al. (2008b) to infer ages for the stars in our sample. We refer the reader to the original work for details; in summary, from a synthetic CMD created with an SFH and chemical enrichment law sampling a broad age and metallicity range $(13 \leq$ age $[\mathrm{Gyr}]<0$ and $-2.5 \leq[\mathrm{Fe} / \mathrm{H}] \leq+0.5)$, the authors obtained a general relation between age, dereddened $(V-I)_{0}$ colour, $V$-band absolute magnitude $M_{V}$ and metallicity for RGB and asymptotic giant branch stars fainter than the RGB tip: ${ }^{7}$

$$
\begin{aligned}
\log _{10}(\text { age })= & 2.57+9.72 \times(V-I)_{0}+0.70 \times M_{V}-1.51 \\
& \times[\mathrm{Fe} / \mathrm{H}]+3.86 \times(V-I)_{0}^{2}-0.19 \\
& \times[\mathrm{Fe} / \mathrm{H}]^{2}+0.49 \times(V-I)_{0}^{3} .
\end{aligned}
$$

The distance modulus and reddening we used to obtain absolute magnitudes and correct $V-I$ colours are listed in Table 1 .

For each star, we first computed $10^{4}$ random realizations of its $(V-I)$ colour, $M_{V}$ and $[\mathrm{Fe} / \mathrm{H}]$, extracting them from a Gaussian probability distribution centred on the observed values and with

\footnotetext{
${ }^{7}$ The synthetic CMD was created with IAC-star (Aparicio \& Gallart 2004) and the BaSTi stellar evolution library (Pietrinferni et al. 2004).
}

$\sigma$ corresponding to the measurement error for each parameter. We then obtain a distribution of ages, by applying equation (4) to these $10^{4}$ random realisations, and adopt as age and its uncertainty for each star the mean and the standard deviation of the distribution. To avoid the regime of saturation of equation (4), which occurs at $\gtrsim 12.5 \mathrm{Gyr}$, as well as unrealistic ages such as younger than that of the youngest stars in the upper part of the RGB, $\sim 1 \mathrm{Gyr}$, we assign a random value within the uncertainty to those objects with ages falling beyond these extremes.

The metallicity as a function of age is shown in the top panel of Fig. 12. The metallicity is found to be $[\mathrm{Fe} / \mathrm{H}] \sim-2.0$ dex at the oldest ages, $\gtrsim 12$ Gyr ago, and, after a slight increase, it remains more or less constant at $[\mathrm{Fe} / \mathrm{H}] \sim-1.6$ until about $4 \mathrm{Gyr}$ ago. Finally, a new increase of metallicity is observed in the last $2 \mathrm{Gyr}$, where $[\mathrm{Fe} / \mathrm{H}]$ reaches up to $\sim-0.5 \mathrm{dex}$.

Our findings are in fairly good agreement with the age-metallicity relation derived from the Phx SFH by Hidalgo et al. (2009), of which we reproduce the main traits in Fig. 12. In the latter, the increase of metallicity at the beginning of the galaxy life is not observed, possibly because of the lack of age resolution in the SFH determination at these old ages. The colour of RGB stars scales linearly with $Z$ and the isochrones become degenerate in metallicity at low $[\mathrm{Fe} / \mathrm{H}]$. Between $\sim 9$ and 5 Gyr ago, the agreement of the age-metallicity relation derived from the CMD with the spectroscopic measurements is excellent. The differences observed for ages younger than $4 \mathrm{Gyr}$ follow from the fact that an upper limit for the metallicity of $[\mathrm{Fe} / \mathrm{H}]$ $\sim-1$ was assumed in the SFH derivation by Hidalgo et al. (2009) and thus the flat profile at young ages in the photometric case, while in the spectroscopic determinations, one can notice a clear increase.

It should be noted that equation (4) was derived assuming an 'MW-like' trend for $[\alpha / \mathrm{Fe}]$ versus $[\mathrm{Fe} / \mathrm{H}]$ :

$[\alpha / \mathrm{Fe}]=\left\{\begin{array}{ccc}0.4 & \text { if } & {[\mathrm{Fe} / \mathrm{H}] \leq-1.0} \\ -0.4 \times[\mathrm{Fe} / \mathrm{H}] & \text { if } & {[\mathrm{Fe} / \mathrm{H}]>-1.0}\end{array}\right.$,

which needs not be appropriate for a dwarf galaxy system in the mass range of $\mathrm{Phx}$, where the 'knee' of $[\alpha / \mathrm{Fe}]$ is likely to occur at much lower metallicities than for the MW (see Tolstoy et al. 2009, for examples of the different trends exhibited by dwarf galaxies). On the other hand, the photometric agemetallicity relation was obtained by using solar-scaled models, while a plateau of $\alpha$-enhancement at low metallicities might have been expected. While the actual chemical properties of Phx are likely to lie somewhere in between these assumptions, it is reasonable to think that the observed differences might partially be due to the adopted $[\alpha / \mathrm{Fe}]$ trends. Hence, overall, we deem the comparison satisfactory.

The lower panel of Fig. 12 shows the age distribution inferred for the stars in our spectroscopic sample. Note that this age distribution cannot be directly compared with the SFH of the galaxy, as derived for example by Hidalgo et al. (2009). The SFH, by definition, provides the information on the amount of mass transformed into stars at a given time, while the fraction of this mass present in alive stars of given age decreases strongly towards older ages. In a random sample of RGB stars near the RGB tip, the number of old stars is underestimated relative to the number of young stars by a few tens of per cent due to the varying lifetime of RGB stars as a function of mass (Cole et al. 2009). Thus, the true age distribution is likely to be suppressed at the young end and enhanced at the old end compared to the shown histogram. 


\subsection{Discrete analytic chemical enrichment models}

Modelling the MDF of dwarf galaxies has significantly increased our understanding of their chemical enrichment: e.g. the shape of the MDF of MW dSph satellites strongly points to a loss of 96 per cent to $>99$ per cent of the metals they have produced (e.g. Kirby, Martin \& Finlator 2011b).

In order to interpret the metallicity properties of a galaxy in terms of its chemical evolution, we need to retrieve the shape of its global MDF. In systems that display metallicity gradients, the observed MDF may suffer from biases, depending on the details of the observing strategy. In the specific case of our slit mask observations of Phx, the spatial distribution of the spectroscopically observed member stars is relatively uniform with radius, unlike the underlying stellar population that is best described by a Sérsic profile (Battaglia et al. 2012). Coupled to the observed strong metallicity gradient, this may cause our sample to miss many of the centrally concentrated metal-rich stars, while overrepresenting the more metal-poor stars that have a more extended spatial distribution. We note that the opposite might happen in systems with strong metallicity gradients where only the central regions have been surveyed.

In this work, we adopt the Kirby et al. (2011a) analytic models to investigate how inflow and outflow of gas have affected the star formation in $\mathrm{Phx}$, but we introduce few important modifications to account for the spatial sampling of the spectroscopic data set with respect to the underlying stellar density.

First, we consider the pristine (leaky box) model that has no gas inflow and where the SNe yields and gas outflow are controlled by a single parameter - the effective yield $(p)$.

We then include the dependence on the elliptical radial distance from the centre of the galaxy, $r$, by considering the $2 \mathrm{D}$ probability distribution function:

$P([\mathrm{Fe} / \mathrm{H}], \mathrm{r}) \propto 10^{[\mathrm{Fe} / \mathrm{H}]} \exp \left(-\frac{10^{[\mathrm{Fe} / \mathrm{H}]}}{\mathrm{p}(\mathrm{r})}\right)$.

The effective yield parameter in units of $Z / Z_{\odot}$ is radially dependent, assuming a linear dependence in $[\mathrm{Fe} / \mathrm{H}]$ space (exponential in $Z$ space):

$p(r)=p_{0} 10^{r \Delta[\mathrm{Fe} / \mathrm{H}]}$,

where $p_{0}$ is the central effective yield and $\Delta[\mathrm{Fe} / \mathrm{H}]$ is the metallicity gradient. The radial dependence of the effective yield parameter could be interpreted as the $\mathrm{SNe}$ yields being better retained in the central regions of the galaxy and more efficiently expelled from the outer parts, where the gravitational potential is shallower. Schroyen et al. (2013) showed that metallicity gradients are robust over time in dwarf galaxies, where star formation proceeds in more and more central regions as time passes, and are not destroyed via radial migration of stars, which happens in large spiral galaxies, for instance.

The model with gas inflow was first introduced by Lynden-Bell (1975) and also used by Kirby et al. (2011a) to show that it describes well the central MDF of some dSphs (e.g. Fornax, Leo I, Draco). In this framework, the galaxy continuously accretes decreasing amount of metal-free gas that is available for star formation. Additionally to the effective yield, this family of models is also characterized by a second parameter $(M>1)$ that describes the accreted gas mass. If $g$ is the mass of the available gas in the galaxy as a fraction of the initial gas mass and $s$ is the stellar mass in the same units then:

$g(s)=\left(1-\frac{s}{M}\right)\left(1+s-\frac{s}{M}\right)$

In the ideal case, where all the gas is converted into stars, $M$ is equal to the final stellar mass as a fraction of the initial gas mass. In the case when $M=1$, there is no extra gas and the model reduces to the pristine case. The only modification to the probability distribution function presented in Kirby et al. (2011a) is that we again assumed radially dependent effective yield in the same form as in equation (7). The extra gas model in our case has three free parameters: $p_{0}, \Delta[\mathrm{Fe} / \mathrm{H}]$ and $M$. The MDF is given by the following two equations:

$$
\begin{aligned}
{[\mathrm{Fe} / \mathrm{H}](s)=} & \log \left(p(r)\left(\frac{M}{1+s-\frac{s}{M}}\right)^{2}\right. \\
& \left.\times\left(\ln \frac{1}{1-\frac{s}{M}}-\frac{s}{M}\left(1-\frac{1}{M}\right)\right)\right)
\end{aligned}
$$

$$
\begin{aligned}
& P([\mathrm{Fe} / \mathrm{H}], r) \\
& \propto \frac{10^{[\mathrm{Fe} / \mathrm{H}]}}{p(r)} \frac{1+s\left(1-\frac{1}{M}\right)}{\left(1-\frac{s}{M}\right)^{-1}-2\left(1-\frac{1}{M}\right) \times 10^{[\mathrm{Fe} / \mathrm{H}]} / p(r)},
\end{aligned}
$$

where we solve equation (9) numerically for $s$ and use the solution in equation (10).

Our likelihood function takes the form:

$$
\begin{aligned}
& \mathscr{L}\left(p_{0}, \Delta[\mathrm{Fe} / \mathrm{H}],(M) \mid[\mathrm{Fe} / \mathrm{H}]_{i}, \delta[\mathrm{Fe} / \mathrm{H}]_{i}, r_{i}\right) \\
& =\prod_{i} \int_{-\infty}^{+\infty} P\left([\mathrm{Fe} / \mathrm{H}], r_{i}\right) \times S\left(r_{i}\right) \times r_{i} \\
& \quad \times \frac{1}{\sqrt{2 \pi} \delta[\mathrm{Fe} / \mathrm{H}]_{i}} \exp \left(-\frac{\left([\mathrm{Fe} / \mathrm{H}]-[\mathrm{Fe} / \mathrm{H}]_{i}\right)^{2}}{2\left(\delta[\mathrm{Fe} / \mathrm{H}]_{i}\right)^{2}}\right) \mathrm{d}[\mathrm{Fe} / \mathrm{H}],
\end{aligned}
$$

where $S\left(r_{i}\right)$ is the Sérsic probability at the position of each star and $[\mathrm{Fe} / \mathrm{H}]_{i}$ and $\delta[\mathrm{Fe} / \mathrm{H}]_{i}$ denote the metallicity and the metallicity uncertainty of each star, respectively. The parameters of the Sérsic profile that best describes the galaxy are fixed from the photometric stellar density profile (see Table 1).

To find the best set of parameters, we maximize the loglikelihood function $(\hat{\mathscr{L}}=\log \mathscr{L})$ using the Metropolis-Hastings Markov Chain Monte Carlo algorithm (Hastings 1970).

Before turning to the observations, we tested the ability of our method to recover the global MDF by simulating mock data of similar size and spatial sampling as for our Phx spectroscopic sample. This is illustrated in Fig. 13. The creation of the mock data samples is as follows: first we create a mock galaxy with $10^{4}$ stars that follow a Sérsic distribution; then we randomly assign a metallicity to each star drawn from our 2D probability distribution function (equation 6 in the case of the pristine model). The metallicity gradient and effective yield parameters were chosen to be similar to what we find in Phx, but in the extra gas case, we decided to use $M=2$ to make it distinct from the pristine model. The global MDF of the 'mock galaxy' is presented with the blue histogram in Fig. 13.

Then we select the stars with positions closest to the positions of the observed Phx stars and assign to them a metallicity uncertainty in agreement with the observed $[\mathrm{Fe} / \mathrm{H}]$ error distribution (Fig. 5), mimicking our target selection in spectroscopic masks. The resulting MDFs (black histograms in the left-hand and middle panels of Fig. 13) are qualitatively similar to the observed MDF of Phx (see the right-hand panel of Fig. 13). The recovered MDF (red lines) are in excellent agreement with the original distributions for both the pristine and extra gas models, notwithstanding the spatially biased and size-limited mock data samples. We made 1000 random realizations of the mock samples as drawn from the pristine and the extra gas models and tried to reconstruct the original distributions. The recovered parameters and resulting MDFs are presented in Fig. 14 for 


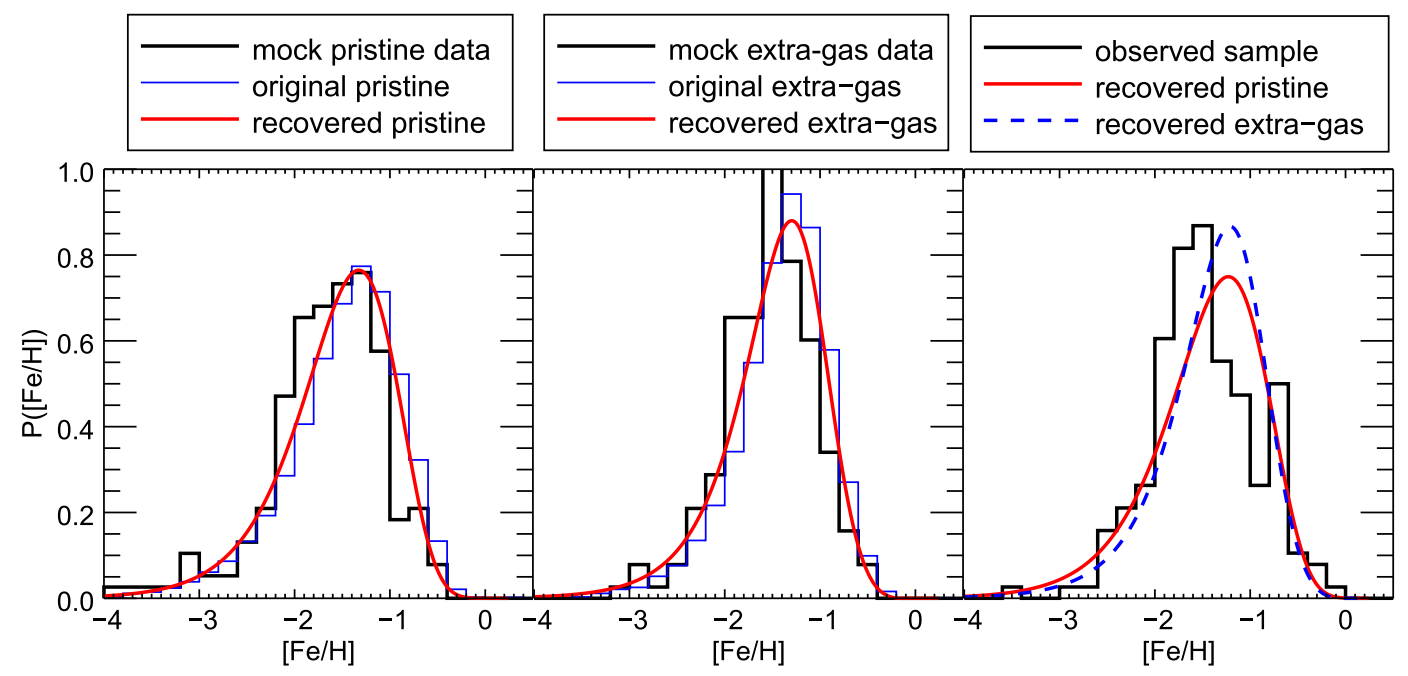

Figure 13. Example mock data and recovered MDF for the pristine (left-hand panel) and the extra gas (middle panel) models. The blue histogram represents the complete mock-galaxy sample, the black histogram represents the mock observations sample that was used to recover the original MDF - red line; right-hand panel: the observed MDF of Phx - black histogram. The recovered full MDFs according to the pristine and extra gas model are overplotted with red and blue dashed lines, respectively.
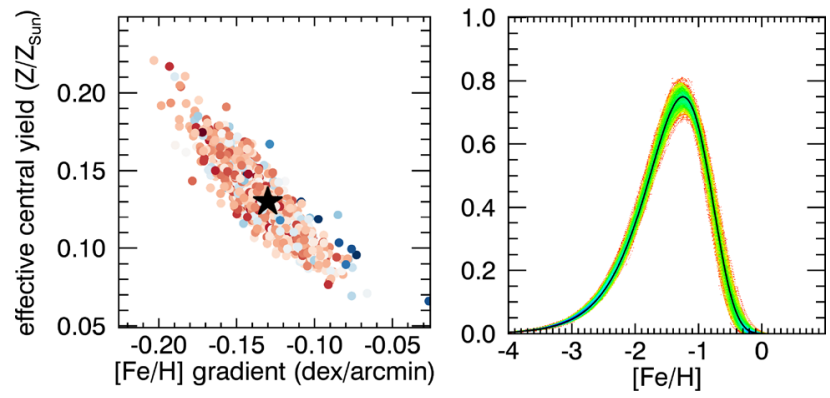

Figure 14. The recovery of the original MDF of 1000 mock data samples taken from the pristine model. The recovered parameters (metallicity gradient and central effective yield) are plotted in the left-hand panel, colour coded by maximum likelihood found during the fit (red - higher likelihood, blue - lower likelihood). The black star indicates the original parameters used to generate the mock data. The resulting MDFs are presented in the right-hand panel, colour coded by density. The original MDF is presented with a thick black line.

the pristine model and Fig. 15 for the extra gas models, respectively. The central effective yield and metallicity gradient parameters appear to be correlated in both models but well centred around the true values. None the less, the trend of metallicity versus radius (Fig. 11) could be used for a direct determination of the metallicity gradient, breaking the degeneracy among the model parameters. In the case of the extra gas model, however, a limited sample of $\sim 190$ stars with fairly large $[\mathrm{Fe} / \mathrm{H}]$ uncertainties does not constrain well enough the $M$ parameter, in general leading to an overestimate. We confirmed that if larger mock samples $(\sim 1000$ stars $)$ with reduced $[\mathrm{Fe} / \mathrm{H}]$ errors are used, then the estimate of the $M$ parameter agrees better with the input value, although is still slightly biased towards higher values.

Fitting the pristine model to the Phx observations, we find $p_{0}=0.13 \pm 0.01$ and $\Delta[\mathrm{Fe} / \mathrm{H}]=-0.13 \pm 0.01 \mathrm{dex} \operatorname{arcmin}^{-1}$, where the quoted uncertainties are the $1 \sigma$ spreads of the parameters in the Markov chain after the burn-in phase. The model estimate of the gradient slope is the same as inferred from the simple linear fit to the run of $[\mathrm{Fe} / \mathrm{H}]$ as a function of radius described in
Section 6.1. In agreement with the pristine model, also with the extra-gas model, we obtain $p_{0}=0.14 \pm 0.01, \Delta[\mathrm{Fe} / \mathrm{H}]=-0.13$ \pm 0.01 ; the parameter quantifying the fraction of accreted gas is $M=1.6 \pm 0.2$; considering that the extra gas parameter tends to be overestimated in our mock samples, we conclude that it is unlikely that Phx accreted large amounts of gas throughout its evolution. When seen in the context of our hypothesis of Phx prolate rotation as possibly caused by a past accretion/merger event, this would imply that the accreted system should not have carried with it a large amount of gas. However, our analysis does not include the youngest stellar population of $\mathrm{Phx}$, which may have been born from a recently captured gas-rich system that is responsible for both the observed prolate rotation and the peculiar distribution of the young stars in a disc/bar like structure perpendicular to the morphology of the old population.

The resulting total MDFs from the two models are presented in the right-hand panel of Fig. 13. In both cases, the recovered MDF peaks at slightly higher $[\mathrm{Fe} / \mathrm{H}]$ than the observed one. One should keep in mind, of course, that the reconstructed MDFs are only realistic if the galaxy does indeed follow one of those empirical enrichment models. While Kirby et al. (2011a) did show that some dSphs' MDFs can be well approximated with these models, there are also notable exceptions in their sample like Sculptor and Leo II. We also attempted to correct the observed MDF for spatial sampling without having to assume a model for the MDF, simply by assigning a weight to each spectroscopic member as in Larsen, Strader \& Brodie (2012), i.e. calculating the ratio between the number of stars predicted by the Sérsic model and spectroscopically observed per bins of elliptical radius. Reassuringly, the resulting MDF shares the same features as those reconstructed from the analytical models, where the contribution of the metal-poor tail decreases and the $[\mathrm{Fe} / \mathrm{H}]$ value of the peak increases with respect to the observed MDF, although it is considerably noisier because of the relatively low number statistics.

Kirby et al. (2013) used the weighted mean $[\mathrm{Fe} / \mathrm{H}]$ to place galaxies on the luminosity-metallicity relation. Assuming a stellar mass $M_{*}=0.77 \times 10^{6} \mathrm{M}_{\odot} \quad($ McConnachie 2012, and references therein), Phx fits well in the Kirby et al. (2013) 

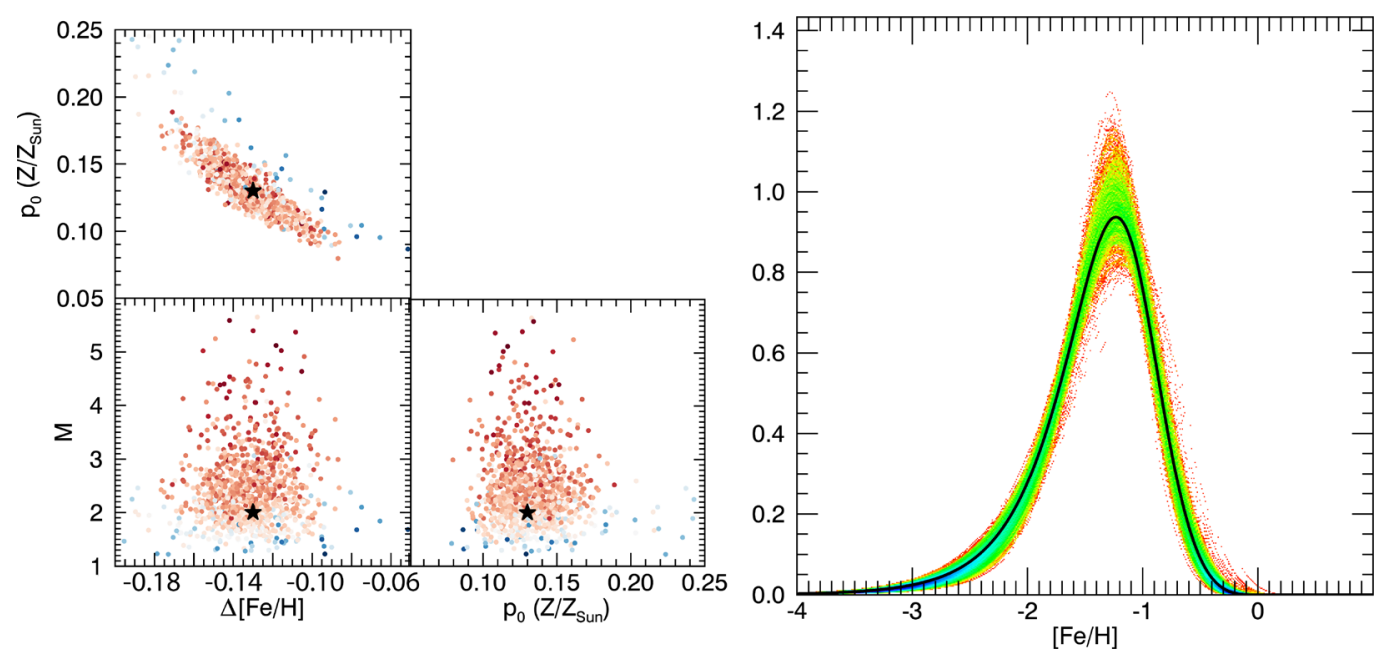

Figure 15. The recovery of the original MDF of 1000 mock data samples taken from the extra gas model. The recovered parameters $\left(\Delta[\mathrm{Fe} / \mathrm{H}], p_{0}, M\right)$ are plotted in the left-hand panel, colour coded by maximum likelihood found during the fit (red - higher likelihood, blue - lower likelihood). The black stars indicate the original parameters used to generate the mock data. The resulting MDFs are presented in the right-hand panel, colour coded by density. The original MDF is presented with a thick black line.

luminosity-metallicity relation, both when we use a mean metallicity $<[\mathrm{Fe} / \mathrm{H}]>-1.49 \pm 0.04$ derived from the observed $\mathrm{MDF}$ and the inferred global $\mathrm{MDF}(<[\mathrm{Fe} / \mathrm{H}]>-1.47$ and -1.41 from the pristine and extra-gas model, respectively).

Finally, we note that the recovered MDF is valid for the RGB but not necessarily for the entire galaxy. As mentioned earlier, the different time that stars of different mass spend on the RGB introduces a bias in the sense that the observed number of red giants does not accurately reflect the actual relative mass of metals. The net effect for a metal-poor, mostly old galaxy like Phx is that the MDF inferred from RGB observations is inflated at the highmetallicity end and suppressed at the low-metallicity end compared to the true underlying MDF. This arises indirectly, because there is a smaller range of stellar mass within the sampled magnitude interval along the RGB for older stars than for younger stars. For the very shallow age-metallicity relation shown by $\mathrm{Phx}$, the effect on the MDF is therefore likely to be small, of the order of 10-20 percent or less per metallicity bin (Manning, private communication). A more quantitative estimate requires careful modelling (Manning \& Cole, in preparation), which exceeds the scope of this work.

\subsection{An extremely metal-poor star candidate}

The study of the detailed chemical abundances of extremely metalpoor $(\mathrm{EMP},[\mathrm{Fe} / \mathrm{H}]<-3)$ stars is considered as particularly insightful for understanding the early chemical enrichment of galaxies as well as the properties of the first stars (Frebel \& Norris 2015).

Among the Local Group dwarf galaxies, so far, the detection of EMP stars has mostly been limited to the dwarf galaxies satellites of the MW (e.g. Frebel et al. 2010; Tafelmeyer et al. 2010; Kirby \& Cohen 2012; Starkenburg et al. 2013), being easier to spot in those dwarfs with the lowest mean metallicities. While their number has been significantly increasing in the last few years, they remain fairly scarce, because searching for them requires either the acquisition of large data sets or selection methods aimed at isolating suitable candidates from the overall population followed by high-resolution spectroscopic follow-up (see e.g. Simon et al. 2015).
Here, we report on an EMP star candidate in our data set. The EMP candidate has a metallicity according to the CaT calibration of $[\mathrm{Fe} / \mathrm{H}]=-3.5 \pm 0.1 \mathrm{dex}$. We measured a heliocentric velocity of $-16.5 \pm 5.8 \mathrm{~km} \mathrm{~s}^{-1}$. The star is found well within the tidal radius of $\mathrm{Phx}$, about 2.8 arcmin from the centre. With a magnitude $V=20.54 \pm 0.04 \mathrm{mag}$ and colour $V-I=1.31 \mathrm{mag}$, it is among the brightest stars in our sample $\left(\mathrm{SNR}=65 \mathrm{px}^{-1}\right.$, very good quality spectrum) and is situated right at the tip of the RGB of Phx. The star can be identified in Fig. 11 as the one with the darkest blue colour. It has by far the weakest $\mathrm{CaT}$ lines in the entire sample with $\mathrm{EW}_{2}=0.72 \pm 0.04$ and $\mathrm{EW}_{3}=0.69 \pm 0.05 \AA$. For comparison, the second most metal-poor star with similar luminosity $V=20.69$ \pm 0.04 mag has $\mathrm{EW}_{2}=1.59 \pm 0.15$ and $\mathrm{EW}_{3}=1.54 \pm 0.12 \AA$ and estimated $[\mathrm{Fe} / \mathrm{H}]=-2.4 \pm 0.1 \mathrm{dex}$. The EW of the $\mathrm{Mg}$ I line at $8806.8 \AA$ is $0.1 \AA$, a value compatible with the object being an EMP star (Battaglia \& Starkenburg 2012).

We performed spectral synthesis using FERgE $^{8}$ (Allende Prieto et al. 2006). To this end, we used a grid of synthetic spectra computed with ASSET (Koesterke, Allende Prieto \& Lambert 2008) from the ATLAS9 model atmospheres (Castelli \& Kurucz 2004). In this case, we used a grid with five dimensions, $[\mathrm{Fe} / \mathrm{H}],[\alpha / \mathrm{Fe}], \log v_{t}$ (microturbulence velocity), $T_{\text {eff }}$ and $\log g$. Other abundances such as $\mathrm{C}$ were fixed to the Solar values. The best-fitting model predicts $[\mathrm{Fe} / \mathrm{H}]=-3.89 \pm 0.15 \mathrm{dex},[\alpha / \mathrm{Fe}]=0.56 \pm 0.30 \mathrm{dex}, T_{\text {eff }}=3696$ $\pm 65 \mathrm{~K}$ and $\log g=1.7 \pm 0.4 \mathrm{dex}$.

An independent spectral synthesis of the object, kindly performed by M. Beasley with PPXF (Cappellari \& Emsellem 2014) with the synthetic spectral models used in Starkenburg et al. (2010), favours a slightly higher $[\mathrm{Fe} / \mathrm{H}]=-3.0$, effective temperature $4700 \mathrm{~K}$, $[\alpha / \mathrm{Fe}]=0.4$ and $\log g=2.2 \mathrm{dex}$.

The three independent methods point to this star being an EMP giant star. Finally, the Besançon model predicts the contamination to be almost entirely due to MW dwarf stars from the halo and thick disc, hence it is unlikely the object is a giant star belonging to the MW.

We conclude that it is highly likely that we have uncovered the presence of an EMP giant star in Phx.

\footnotetext{
${ }^{8}$ http://vivaldi.ll.iac.es/galeria/callende/ferre/
} 


\section{CONCLUSIONS}

In this work, we presented results from our VLT/FORS2 multiobject spectroscopy of 280 targets, 196 of which were identified as very likely RGB stars, members of the Phx dwarf galaxy. These data are used to characterize the internal, wide-area kinematic and metallicity properties of this system and represent the first spectroscopic metallicity measurements available for this galaxy. We also present a modification to the analytical chemical enrichment models of Kirby et al. (2011a) that accounts for metallicity gradients and resulting observational biases.

$\mathrm{Phx}$ is approaching the MW with a heliocentric (GSR) velocity of $-21.2 \pm 1.0 \mathrm{~km} \mathrm{~s}^{-1}\left(-108.6 \pm 1.0 \mathrm{~km} \mathrm{~s}^{-1}\right)$. Our determination confirms the systemic velocity measurement by Irwin \& Tolstoy (2002) and re-enforces the hypothesis of a physical association between Phoenix and the nearby $\mathrm{H}$ I cloud moving at $-23 \mathrm{~km} \mathrm{~s}^{-1}$ (St-Germain et al. 1999).

The analysis of the internal kinematic properties reveals the presence of a velocity gradient along the projected minor axis. Since we can exclude perspective effects as possible causes of the observed gradients, we deem it as highly likely to have detected prolate rotation in this system. Interestingly, the rotation signal is aligned with the spatial distribution of young stars, known to be almost perpendicular to the spatial distribution of Phoenix main body. There is only one other Local Group dwarf galaxy known to display prolate rotation, And II, likely caused by a dwarf-dwarf merger or accretion (Amorisco et al. 2014). We speculate that also Phoenix might have experienced a similar event in the past, giving rise both to the prolate rotation and the peculiar distribution of young stars.

As in other dwarf galaxies of similar luminosity, the MDF of Phx RGB stars spans almost 3 dex in $[\mathrm{Fe} / \mathrm{H}]$. When corrected for the spatial sampling of the spectroscopic data set, the mean $[\mathrm{Fe} / \mathrm{H}]$ $=-1.47 \mathrm{dex}$ from the resulting MDF would place Phx in general agreement with the $[\mathrm{Fe} / \mathrm{H}]-\mathrm{L}_{V}$ relation defined by other Local Group galaxies (Kirby et al. 2013).

We find a clear negative metallicity gradient that can be approximated by a linear relation with a slope $0.13 \pm 0.01 \mathrm{dex} \operatorname{arcmin}^{-1}$. Similarly steep gradients have been observed in most MW dSphs of luminosity comparable to Phx. The fact that such steep metallicity gradients are present in dwarf galaxies inhabiting a dense environment (i.e. satellites of the MW) and in a system found beyond the MW virial radius and likely at its first approach towards the MW points towards the conclusion that metallicity gradients are an intrinsic property of these low-luminosity galaxies.

Wide-area spectroscopic surveys of Local Group dwarf galaxies continue unveiling surprising characteristics of these systems at the low-mass end of the galaxy mass function and provide insights into the mechanisms driving the evolution of this numerous galaxy population.

\section{ACKNOWLEDGEMENTS}

This paper is based on observations made with ESO telescopes at the La Silla Paranal Observatory under programme IDs 083.B0252(B) and 71.B-0516. We thank the referee Evan Kirby for his positive and constructive report. We thank Michael Hilker for his help with the data reduction process, Mike Beasley for performing the spectral synthesis of the EMP candidate star and Ryan Leaman for kindly providing the metallicity gradients of MW dSphs and gasrich objects shown in Fig. 11. We thank Glenn van de Ven, Morgan Fouesneau and Ryan Leaman for insightful discussions on the contents of this study. This study was partially financially supported by a 2015 ESO DGDF grant. NK acknowledges financial support from IAC for a three-week visit to the institute. GB gratefully acknowledges support through a Marie- Curie action Intra European Fellowship, funded by the European Union Seventh Framework Program (FP7/2007-2013) under Grant agreement number PIEFGA-2010-274151, as well as the financial support by the Spanish Ministry of Economy and Competitiveness (MINECO) under the Ramon y Cajal Programme (RYC-2012-11537). AC was supported by a fellowship from the Netherlands Research School for Astronomy (NOVA). This work made extensive use of the NASA Astrophysics Data System bibliographic services. This research used the facilities of the Canadian Astronomy Data Centre operated by the National Research Council of Canada with the support of the Canadian Space Agency.

\section{REFERENCES}

Allende Prieto C., Beers T. C., Wilhelm R., Newberg H. J., Rockosi C. M., Yanny B., Lee Y. S., 2006, ApJ, 636, 804

Amorisco N. C., Evans N. W., van de Ven G., 2014, Nature, 507, 335

Aparicio A., Gallart C., 2004, AJ, 128, 1465

Appenzeller I. et al., 1998, The Messenger, 94, 1

Armandroff T. E., Da Costa G. S., 1991, AJ, 101, 1329

Armandroff T. E., Zinn R., 1988, AJ, 96, 92

Battaglia G., Starkenburg E., 2012, A\&A, 539, A123

Battaglia G. et al., 2006, A\&A, 459, 423

Battaglia G., Irwin M., Tolstoy E., Hill V., Helmi A., Letarte B., Jablonka P., 2008a, MNRAS, 383, 183

Battaglia G., Helmi A., Tolstoy E., Irwin M., Hill V., Jablonka P., 2008b, ApJ, 681, L13

Battaglia G., Tolstoy E., Helmi A., Irwin M., Parisi P., Hill V., Jablonka P., 2011, MNRAS, 411, 1013

Battaglia G., Rejkuba M., Tolstoy E., Irwin M. J., Beccari G., 2012, MNRAS, 424,1113

Binney J., Tremaine S., 1987, Galactic Dynamics, Princeton Univ. Press, Princeton, NJ

Bovy J., Hogg D. W., Rix H.-W., 2009, ApJ, 704, 1704

Boylan-Kolchin M., Bullock J. S., Sohn S. T., Besla G., van der Marel R. P., 2013, ApJ, 768, 140

Cappellari M., Emsellem E., 2014, Publ. Astron. Soc. Pac., 116, 138

Carrera R., 2012, A\&A, 544, A109

Carrera R., Gallart C., Hardy E., Aparicio A., Zinn R., 2008a, AJ, 135, 836

Carrera R., Gallart C., Aparicio A., Costa E., Méndez R. A., Noël N. E. D., 2008b, AJ, 136, 1039

Carrera R., Pancino E., Gallart C., del Pino A., 2013, MNRAS, 434, 1681

Castelli F., Kurucz R. L., 2004, preprint (arXiv:e-prints)

Cayrel R., 1988, in Cayrel de Strobel G., Spite M., eds, Proc. IAU Symp. 132, The Impact of Very High S/N Spectroscopy on Stellar Physics. Kluwer, Dordrecht, p. 345

Cenarro A. J., Cardiel N., Gorgas J., Peletier R. F., Vazdekis A., Prada F., 2001, MNRAS, 326, 959

Cole A. A., Smecker-Hane T. A., Tolstoy E., Bosler T. L., Gallagher J. S., 2004, MNRAS, 347, 367

Cole A. A., Tolstoy E., Gallagher J. S., III, Smecker-Hane T. A., 2005, AJ, 129,1465

Cole A. A., Grocholski A. J., Geisler D., Sarajedini A., Smith V. V., Tolstoy E., 2009, in Van Loon J. T., Oliveira J. M., eds, Proc. IAU Symp. 256, The Magellanic System: Stars, Gas, and Galaxies. Kluwer, Dordrecht, p. 263

Coleman M. G., Da Costa G. S., Bland-Hawthorn J., Freeman K. C., 2005 AJ, 129, 1443

Collins M. L. M. et al., 2013, ApJ, 768, 172

de Boer T. J. L. et al., 2012a, A\&A, 539, A103

de Boer T. J. L. et al., 2012b, A\&A, 544, A73

De Rijcke S., Dejonghe H., Zeilinger W. W., Hau G. K. T., 2004, A\&A, 426,53 
Dehnen W., Binney J., 1998, MNRAS, 294, 429

Emsellem E. et al., 2004, MNRAS, 352, 721

Faria D., Feltzing S., Lundström I., Gilmore G., Wahlgren G. M., Ardeberg A., Linde P., 2007, A\&A, 465, 357

Fouquet S., Lokas E. L., del Pino A., Ebrova I., 2017, MNRAS, 464, 2717

Fraternali F., Tolstoy E., Irwin M. J., Cole A. A., 2009, A\&A, 499, 121

Frebel A., Norris J. E., 2015, ARA\&A, 53, 631

Frebel A., Simon J. D., Geha M., Willman B., 2010, ApJ, 708, 560

Gallart C., Martínez-Delgado D., Gómez-Flechoso M. A., Mateo M., 2001, AJ, 121, 2572

Gallart C. et al., 2015, ApJ, 811, L18

Gebhardt K., Pryor C., O’Connell R. D., Williams T. B., Hesser J. E., 2000, AJ, 119, 1268

Gwinn C. R., Moran J. M., Reid M. J., 1992, ApJ, 393, 149

Harris W. E., 1996, AJ, 112, 1487

Hastings W. K., 1970, Biometrika, 57, 97

Hendricks B., Koch A., Walker M., Johnson C. I., Peñarrubia J., Gilmore G., 2014a, A\&A, 572, A82

Hendricks B., Koch A., Lanfranchi G. A., Boeche C., Walker M., Johnson C. I., Peñarrubia J., Gilmore G., 2014b, ApJ, 785, 102

Hidalgo S. L., Aparicio A., Martínez-Delgado D., Gallart C., 2009, ApJ, 705,704

Hidalgo S. L. et al., 2013, ApJ, 778, 103

Ho N. et al., 2012, ApJ, 758, 124

Ho N., Geha M., Tollerud E. J., Zinn R., Guhathakurta P., Vargas L. C., 2015, ApJ, 798, 77

Holtzman J. A., Smith G. H., Grillmair C., 2000, AJ, 120, 3060

Irwin M., Tolstoy E., 2002, MNRAS, 336, 643

Kirby E. N., Cohen J. G., 2012, AJ, 144, 168

Kirby E. N., Lanfranchi G. A., Simon J. D., Cohen J. G., Guhathakurta P., 2011a, ApJ, 727, 78

Kirby E. N., Martin C. L., Finlator K., 2011b, ApJ, 742, L25

Kirby E. N., Cohen J. G., Guhathakurta P., Cheng L., Bullock J. S., Gallazzi A., 2013, ApJ, 779, 102

Kirby E. N., Bullock J. S., Boylan-Kolchin M., Kaplinghat M., Cohen J. G., 2014, MNRAS, 439, 1015

Kleyna J., Wilkinson M. I., Evans N. W., Gilmore G., Frayn C., 2002, MNRAS, 330, 792

Koch A., Grebel E. K., Wyse R. F. G., Kleyna J. T., Wilkinson M. I., Harbeck D. R., Gilmore G. F., Evans N. W., 2006, AJ, 131, 895

Koch A., Wilkinson M. I., Kleyna J. T., Gilmore G. F., Grebel E. K., Mackey A. D., Evans N. W., Wyse R. F. G., 2007, ApJ, 657, 241

Koch A., McWilliam A., Grebel E. K., Zucker D. B., Belokurov V., 2008, ApJ, 688, L13

Koesterke L., Allende Prieto C., Lambert D. L., 2008, ApJ, 680, 764

Lane R. R., Kiss L. L., Lewis G. F., Ibata R. A., Siebert A., Bedding T. R., Székely P., Szabó G. M., 2011, A\&A, 530, A31

Larsen S. S., Strader J., Brodie J. P., 2012, A\&A, 544, L14

Leaman R., Cole A. A., Venn K. A., Tolstoy E., Irwin M. J., Szeifert T., Skillman E. D., McConnachie A. W., 2009, ApJ, 699, 1

Leaman R. et al., 2013, ApJ, 767, 131

Lewis G. F., Ibata R. A., Chapman S. C., McConnachie A., Irwin M. J., Tolstoy E., Tanvir N. R., 2007, MNRAS, 375, 1364

Łokas E. L., Ebrová I., Pino A. d., Semczuk M., 2014, MNRAS, 445, L6

Lynden-Bell D., 1975, Vistas Astron., 19, 299

McConnachie A. W., 2012, AJ, 144, 4

McConnachie A. W., Irwin M. J., 2006, MNRAS, 365, 1263

Markwardt C. B., 2009, in Bohlender D. A., Durand D., Dowler P., eds, ASP Conf. Ser. Vol. 411, Astronomical Data Analysis Software and Systems XVIII. Astron. Soc. Pac., San Francisco, p. 251

Martínez-Delgado D., Gallart C., Aparicio A., 1999a, AJ, 118, 862

Martínez-Delgado D., Gallart C., Aparicio A., 1999b, AJ, 118, 862

Mateo M. L., 1998, ARA\&A, 36, 435

Mayer L., Mastropietro C., Wadsley J., Stadel J., Moore B., 2006, MNRAS, 369,1021
Muñoz R. R. et al., 2005, ApJ, 631, L137

Pietrinferni A., Cassisi S., Salaris M., Castelli F., 2004, ApJ, 612, 168

Robin A. C., Reylé C., Derrière S., Picaud S., 2003, A\&A, 409, 523

Rutledge G. A., Hesser J. E., Stetson P. B., 1997, Publ. Astron. Soc. Pac., 109, 907

Schechter P. L., Gunn J. E., 1978, AJ, 83, 1360

Schönrich R., Binney J., Dehnen W., 2010, MNRAS, 403, 1829

Schroyen J., de Rijcke S., Valcke S., Cloet-Osselaer A., Dejonghe H., 2011, MNRAS, 416, 601

Schroyen J., De Rijcke S., Koleva M., Cloet-Osselaer A., Vandenbroucke B., 2013, MNRAS, 434, 888

Simon J. D., Jacobson H. R., Frebel A., Thompson I. B., Adams J. J., Shectman S. A., 2015, ApJ, 802, 93

St-Germain J., Carignan C., Côte S., Oosterloo T., 1999, AJ, 118, 1235

Starkenburg E. et al., 2010, A\&A, 513, A34

Starkenburg E. et al., 2013, A\&A, 549, A88

Strigari L. E., Frenk C. S., White S. D. M., 2010, MNRAS, 408, 2364

Swan J., Cole A. A., Tolstoy E., Irwin M. J., 2016, MNRAS, 456, 4315

Tafelmeyer M. et al., 2010, A\&A, 524, A58

Tolstoy E., Irwin M. J., Cole A. A., Pasquini L., Gilmozzi R., Gallagher J. S., 2001, MNRAS, 327, 918

Tolstoy E. et al., 2004, ApJ, 617, L119

Tolstoy E., Hill V., Tosi M., 2009, ARA\&A, 47, 371

Tonry J., Davis M., 1979, AJ, 84, 1511

Tremaine S., 1981, in Fall S. M., Lynden-Bell D., eds, Structure and Evolution of Normal Galaxies. Cambridge Univ. Press, Cambridge, p. 67

Tully R. B., Shaya E. J., Karachentsev I. D., Courtois H. M., Kocevski D. D., Rizzi L., Peel A., 2008, ApJ, 676, 184

van de Rydt F., Demers S., Kunkel W. E., 1991, AJ, 102, 130

van de Ven G., van den Bosch R. C. E., Verolme E. K., de Zeeuw P. T., 2006, A\&A, 445, 513

van den Bosch R. C. E., van de Ven G., Verolme E. K., Cappellari M., de Zeeuw P. T., 2008, MNRAS, 385, 647

van Dokkum P. G., 2001, PASP, 113, 1420

Vásquez S., Zoccali M., Hill V., Gonzalez O. A., Saviane I., Rejkuba M., Battaglia G., 2015, A\&A, 580, A121

Walker M. G., Mateo M., Olszewski E. W., Bernstein R., Wang X., Woodroofe M., 2006, AJ, 131, 2114

Walker M. G., Mateo M., Olszewski E. W., Sen B., Woodroofe M., 2009, AJ, 137, 3109

Walker M. G., Olszewski E. W., Mateo M., 2015, MNRAS, 448, 2717

Warren S. R., Cole A. A., 2009, MNRAS, 393, 272

Wheeler C. et al., 2015, MNRAS, preprint (arXiv:1511.01095)

Wilkinson M. I., Kleyna J. T., Evans N. W., Gilmore G. F., Irwin M. J., Grebel E. K., 2004, ApJ, 611, L21

Young L. M., Lo K. Y., 1997, ApJ, 490, 710

Young L. M., Skillman E. D., Weisz D. R., Dolphin A. E., 2007, ApJ, 659, 331

Zaggia S., Held E. V., Sommariva V., Momany Y., Saviane I., Rizzi L., 2011, in Koleva M., Prugniel P., Vauglin I., eds, EAS Publ. Ser. Vol. 48, p. 215

\section{SUPPORTING INFORMATION}

Supplementary data are available at MNRAS online.

\section{Phx_CDS.dat}

Please note: Oxford University Press is not responsible for the content or functionality of any supporting materials supplied by the authors. Any queries (other than missing material) should be directed to the corresponding author for the article.

This paper has been typeset from a $\mathrm{T}_{\mathrm{E}} \mathrm{X} / \mathrm{L} \mathrm{T} \mathrm{EX}$ file prepared by the author. 